\title{
A CHARACTERIZATION OF THE MATHIEU GROUP $\mathfrak{M}_{12}$
}

\author{
BY \\ RICHARD BRAUER AND PAUL FONG (1)
}

In this paper we give a characterization of the simple Mathieu group $\mathfrak{M}_{12}$ of order 95,040 . The character table of $\mathfrak{M}_{12}$ was computed by Frobenius [6], and from his results it can be immediately seen that there exists an element $F$ in $\mathfrak{M}_{12}$ of order 8 such that (i) the cyclic subgroup $\langle F\rangle$ generated by $F$ is self-centralizing, (ii) $F$ is conjugate to its odd powers. Elementary arguments show that there is then a Sylow 2-subgroup $\mathfrak{P}$ of $\mathfrak{M}_{12}$, of order 64 , such that (i), (ii) hold in $\mathfrak{P}$. We are thus led to a consideration of 2-groups $\mathfrak{P}$ of order 64 in which (i), (ii) hold, and groups $(5$ containing $\mathfrak{P}$ as a Sylow 2-subgroup. Our main result is the following:

THEOREM (6A). Let $\left(5\right.$ be a finite group of order $64 g^{\prime}$, where $g^{\prime}$ is odd. Suppose there is an element $F$ of order 8 in $\mathbb{G}$ such that $\langle F\rangle$ is self-centralizing in some Sylow 2-subgroup $\mathfrak{P}$, and $F$ is conjugate to its odd powers in $\mathfrak{P}$. Then one of the following possibilities hold:

(a) (5 has a subgroup of index 2.

(b) (5 has one class of involutions.

(c) If $\mathrm{O}_{2},(\mathfrak{5})$ is the maximal normal subgroup of $(5)$ of odd order, then $\mathfrak{5} / \mathrm{O}_{2},(\mathfrak{G}) \simeq \mathfrak{G}_{1344}$ or $\mathfrak{M}_{12}$, where $\mathfrak{G}_{1344}$ is a uniquely determined nonsimple, nonsolvable group of order 1344 , and $\mathfrak{M}_{12}$ is the Mathieu group on 12 symbols.

In particular, the only simple group with more than one class of involutions satisfying the assumptions of the theorem is $\mathfrak{M}_{12}$. A recent characterization of $\mathfrak{M}_{12}$ by Wong [11], where additional assumptions on the centralizer of a center involution were made, is included in the above result.

In $\S 1,2$-groups $\mathfrak{P}$ in which (i), (ii) hold are investigated. It will be shown in $\S 2$ that if such a group $\mathfrak{P}$ is a Sylow subgroup of a group $\mathfrak{G}$ with no subgroups of index 2 , then the structure of $\mathfrak{P}$ is completely determined. The possible distributions of the involutions of $\mathfrak{P}$ into the conjugate classes of $\mathfrak{G}$ fall essentially into three cases I, II, III. In I, $\mathbf{6}$ has one class of involutions; in II, III 65 has two classes of involutions. The $\S \S 4-6$ are largely concerned with cases II, III, which

Received by the editors February 15, 1965.

(1) This research was partially supported by the Air Force contract AF 49 (638)-1381, and by the National Science Foundation contract NSF GP-1610. We would like to thank the referee for his careful reading of the manuscript and for his valuable suggestions. 
correspond to part (c) of the above theorem. Case I is incomplete as yet, but we hope to continue the work later on.

Notation. All groups $\mathfrak{G}$ considered are finite. If $\mathfrak{H}$ is a subgroup of $\mathfrak{5}$, we write $\mathfrak{H} \leqq(\mathfrak{F}$; in case $\mathfrak{H}$ is normal in $\mathfrak{b}$, we write $\mathfrak{H} \leqq(\mathfrak{b}$. If $\mathfrak{U}$ is a subset of $\mathfrak{b}$, then $\mathfrak{A}(\mathfrak{U})$ and $\mathfrak{C}(\mathfrak{U})$ are the normalizer and centralizer of $\mathfrak{A}$ in $\mathfrak{G}$; their orders are respectively $n(\mathfrak{A})$ and $c(\mathfrak{U})$. At times it will be necessary to attach a subscript $\left(\mathfrak{G}\right.$ to $\mathfrak{N}_{\mathfrak{G}}(\mathfrak{U})$ and $\mathfrak{C}_{\mathfrak{G}}(\mathfrak{U})$ when $\mathfrak{U}$ is contained in several groups. The subgroup of $(\mathfrak{5}$ generated by $\mathfrak{A}$ will be denoted by $\langle\mathfrak{U}\rangle$. If $\mathfrak{A}$ consists of only one element $A$, we will write $A$ for $\mathfrak{A}$.

Let $p$ be a fixed rational prime; $v$ will then be the exponential valuation of the rational numbers determined by $p$, normalized by setting $v(p)=1$. If $G \in \mathfrak{G}$, we will write $v(G)$ for $v(c(G)) . G$ is a $p$-element, a $p$-regular element, or a $p$-singular element if $G$ has order a power of $p$, relatively prime to $p$, or divisible by $p$ respectively. Each $G \in\left(5\right.$ is a unique product $G=G_{1} G_{2}$, where $G_{1}$ is a $p$-element, $G_{2}$ is $p$-regular, and each $G_{i}$ is a power of $G . G_{1}$ is called the $p$-factor of $G, G_{2}$ the $p$-regular or $p^{\prime}$-factor of $G$. If $P$ is a $p$-element, then the section $S(P)$ of $P$ is the set $\left\{G \in \mathbb{E} \mid p\right.$-factor of $G$ is conjugate to $P$ in $(5)$. An $S_{p}$-subgroup of $\mathbb{E}$ is a Sylow $p$-subgroup of $\mathfrak{W}$.

If $\mathfrak{P}$ is an $S_{p}$-subgroup of $\mathfrak{G}$, we will denote the $\mathfrak{P}$-conjugate classes by $\operatorname{ccl}(P)$, where $P$ is a representative of the class. At times all the elements of the class will be displayed between the parenthesis signs. If two classes of $\mathfrak{P}$ are conjugate in (5), we will say they are fused in $\mathfrak{5}$. $A$ class of $\mathfrak{P}$ which is not fused to any other $\mathfrak{P}$-class is said to be isolated. The symbol $\sim$ will mean conjugate to. The transform of $A$ by $G$ is $G^{-1} A G=A^{G}$; we write $G: A \rightarrow G^{-1} A G$.

1. Let $\mathfrak{P}$ be a 2-group of order 64 satisfying the following condition: There exists a self-centralizing element $F$ of order 8 which is conjugate in $\mathfrak{P}$ to its odd powers, i.e.

(i) $\mathfrak{C}(F)=\langle F\rangle$,

(ii) $F \sim F^{3} \sim F^{5} \sim F^{7}$.

The determination of the structure of $\mathfrak{P}$ will proceed in several steps.

(a) Let $\mathfrak{N}$ be the normalizer of $\langle F\rangle$. By conditions (i), (ii) $\mathfrak{N}$ has order 32 , and hence $\mathfrak{N} \preccurlyeq \mathfrak{P}$. Moreover, $\mathfrak{N} /\langle F\rangle$ is abelian of type $(2,2)$, since the automorphism group of $Z_{8}$ is noncyclic.

(b) Let the class of $F$ be $\operatorname{ccl}\left(F, F^{3}, F^{5}, F^{7}, F_{1}, F_{1}^{3}, F_{1}^{5}, F_{1}^{7}\right) . \Re$ permutes by transformation the elements of $\operatorname{ccl}(F)$ among themselves. In particular $F_{1}^{-1} F F_{1} \in \operatorname{ccl}(F)$. Condition (i) implies $F_{1}^{-1} F F_{1}=F^{\alpha}$ for some $\alpha$. Thus $F_{1} \in \mathfrak{N}$ and $F_{1}^{2} \in\langle F\rangle$. Replacing $F_{1}$ by $F_{1}^{3}$ if necessary, we may assume that $F_{1}^{2}=F^{2}$. Let $\mathfrak{F}=\left\langle F, F_{1}\right\rangle ; \mathfrak{F}$ has order 16 , and at least 8 elements of order 8 . A check of the groups of order 16 , which are completely known, shows that we may take $\alpha=5$. If we set $X=F_{1} F$, then $\mathfrak{F}$ is the group $\left\langle X, F \mid X^{2}=F^{8}=1, X F X=F^{5}\right\rangle$. The class $\operatorname{ccl}(F)$ can then be written as $\operatorname{ccl}\left(F, F^{3}, F^{5}, F^{7}, X F, X F^{3}, X F^{5}, X F^{7}\right)$. 
The center of $\mathfrak{F}$ is $\left\langle F^{2}\right\rangle$; for notational convenience, set $J=F^{4}$. Since $\mathfrak{F}$ is generated by $\operatorname{ccl}(F), \mathfrak{F} \leq \mathfrak{P}$. It is easily seen that the conjugate classes of $\mathfrak{P}$ in $\mathfrak{F}$ are

$$
\operatorname{ccl}(F), \operatorname{ccl}\left(F^{2}, F^{-2}\right), \operatorname{ccl}(J), \operatorname{ccl}(1), \operatorname{ccl}\left(X F^{2}, X F^{-2}\right), \operatorname{ccl}(X, X J) .
$$

(c) For any $P \in \mathfrak{P}, P: F \rightarrow \operatorname{ccl}(F), X \rightarrow \operatorname{ccl}(X)$. Since there are 8 possible images for $F$ and 2 for $X$, there are in all 16 possible actions for $P$. If $P: F \rightarrow F$, $X \rightarrow X$, then $P$ centralizes $\mathfrak{F}$, and hence belongs to the center $\mathfrak{Z} \mathfrak{F}$ of $\mathfrak{F}$. A comparison of the orders of $\mathfrak{P}$ and $3 \mathfrak{F}$ implies that all 16 possibilities occur. In particular, there exist elements $N$ and $E$ in $\mathfrak{P}$ such that

$$
N: \begin{aligned}
& F \rightarrow F^{-1}, \\
& X \rightarrow X,
\end{aligned} \quad E: \begin{aligned}
& F \rightarrow X F, \\
& X \rightarrow X J .
\end{aligned}
$$

$N \in \mathfrak{N}$, so that $\mathfrak{N}=\langle N, X, F\rangle$. However, $E \notin \mathfrak{N}$, and so $\mathfrak{P}=\langle E, N, X, F\rangle$ $=\langle E, N, F\rangle$.

(d) Now $N^{2}: F \rightarrow F$, and thus $N^{2} \in\langle F\rangle$. Since $N \notin \mathscr{C}\left(F^{2}\right), N^{2}$ must be 1 or $J$. We write $N^{2}=J^{\rho}$, where $\rho=0$ or 1 . Also $E^{2}: F \rightarrow F^{5}, X \rightarrow X$ so that $E^{2} X \in \mathbb{C}(\mathfrak{F})$ or $E^{2} X \in \mathfrak{Z} \mathfrak{F}$. If $E^{2} X=1$ or $J$, then $E \in \mathbb{C}(X)$, which is not the case. We may thus write $E^{2}=X F^{2} J^{\sigma}$, where $\sigma=0$ or 1 . To complete the description of $\mathfrak{P}$, it remains to compute $E^{-1} N E$. Now $E^{-1} N E: X \rightarrow X, F \rightarrow F^{3}$, so that $E^{-1} N E(N X)^{-1} \in \mathbb{C}(\mathfrak{F})$. Hence $E^{-1} N E=F^{2 \tau} N X=N X F^{-2 \tau}$. Since we may replace $E$ by $F^{2} E$ in (1.1), we may assume $\tau=0$ or 1 . We have proved the first part of

Proposition (1A). Let $\mathfrak{P}$ be a 2-group of order 64 such that there exists a self-centralizing element $F$ of order 8 conjugate in $\mathfrak{P}$ to its odd powers. Set $F^{4}=J$. Then $\mathfrak{P}$ has a normal series

$$
\langle F\rangle \unlhd\langle X, F\rangle \triangleq\langle N, X, F\rangle \triangleq\langle E, N, X, F\rangle=\mathfrak{P}
$$

where $X^{2}=1, X F X=F J ; N^{2}=J^{\rho}, N^{-1} X N=X, N^{-1} F N=F^{-1} ; E^{2}=X F^{2} J^{\sigma}$, $E^{-1} N E=N X F^{-2 \tau}, E^{-1} X E=X J, E^{-1} F E=X F$. Here $\rho, \sigma, \tau$ are 0 or 1 . The commutator subgroup $\mathfrak{P}^{\prime}$ of $\mathfrak{P}$ is $\left\langle X, F^{2}\right\rangle ; \mathfrak{P} / \mathfrak{P}^{\prime}$ is of type $(2,2,2)$. The center of $\mathfrak{P}$ is $\langle J\rangle$.

Proof. It is clear from the above relations that $\mathfrak{P}^{\prime} \geqq\left\langle X, F^{2}\right\rangle$, and that $\left\langle X, F^{2}\right\rangle \triangleq \mathfrak{P}$. Modulo $\left\langle X, F^{2}\right\rangle$ the generators $E, N, F$ of $\mathfrak{P}$ commute and have exponent 2. The assertions about $\mathfrak{P}^{\prime}$ and $\mathfrak{P} / \mathfrak{P}^{\prime}$ now follow. $\mathfrak{z} \mathfrak{P}$ must lie in $\langle F\rangle$. Since $N \notin \mathfrak{C}\left(F^{2}\right), \mathfrak{Z} \mathfrak{P}=\langle J\rangle$. This completes the proof. These groups are incicidentally the ones on pp. 222 and 224 of Hall-Senior [7] $\left.{ }^{2}\right)$.

(2) These groups were pointed out to us by Professor P. N. Burgoyne. 
The following remarks are easy consequences of (1A). Each element of $\mathfrak{P}$ is uniquely expressible in the form $E^{i} N^{j} X^{k} F^{m}$, where $i, j, k=0,1$; and $0 \leqq m \leqq 7$. We have

$$
E \rightarrow E X J, \quad E \rightarrow E J, \quad E \rightarrow E X F^{2 \tau}, \quad N \rightarrow N X F^{-2 \tau},
$$

$$
\begin{aligned}
& F: N \rightarrow N F^{2}, \quad X: N \rightarrow N, \quad N: X \rightarrow X, \quad E: X \rightarrow X J, \\
& X \rightarrow X J, \quad F \rightarrow F J, \quad F \rightarrow F^{-1}, \quad F \rightarrow X F .
\end{aligned}
$$

In particular, the subgroups $\left\langle E^{2}, E F\right\rangle,\left\langle F^{2}, E F\right\rangle$ for $\sigma=1$ and the subgroups $\langle N F, X\rangle=\langle N X F, X\rangle,\left\langle N F^{-1}, X\right\rangle=\left\langle N X F^{-1}, X\right\rangle$ are dihedral of order 8 .

For later use it will be necessary to have a complete set of representatives of the conjugate classes of $\mathfrak{P}$, and the centralizers of these representatives. In the tables below the columns contain from left to right (i) a representative of the class, (ii) the elements of the class, (iii) the centralizer of the representative, (iv) the order of the representative, (v) the square of the representative. The calculations for the most part are straightforward; the details are omitted in such cases.

(a) Classes in $\mathfrak{F}$. The results in this case are easily derived from the steps leading up to the proof of (1A).

\begin{tabular}{|c|l|c|c|c|}
\hline$F$ & $F^{2 i+1}, X F^{2 i+1}, 0 \leqq i \leqq 3$ & $\langle F\rangle$ & 8 & $F^{2}$ \\
$F^{2}$ & $F^{2}, F^{-2}$ & $\left\langle\mathfrak{P}^{\prime}, F, E N\right\rangle$ & 4 & $J$ \\
$X F^{2}$ & $X F^{2}, X F^{-2}$ & $\left\langle\mathfrak{P}^{\prime}, E, N F\right\rangle$ & 4 & $J$ \\
$J$ & $J$ & $\mathfrak{P}$ & 2 & 1 \\
$X$ & $X, X J$ & $\left\langle\mathfrak{P}^{\prime}, N, E F\right\rangle$ & 2 & 1 \\
1 & 1 & $\mathfrak{P}$ & 1 & 1 \\
\hline
\end{tabular}

(b) Classes in $\mathfrak{N}-\mathfrak{F}$. Under repeated transformations by $F$ and $E$, we have $N \sim N F^{2 i}, N \sim N X F^{2 i}, N F \sim N F^{2 i+1}, N X F \sim N X F^{2 i+1}$ for $0 \leqq i \leqq 3$. Since $\mathfrak{C}(N) \geqq\langle N, X, J\rangle$ has order $\geqq 8$, the class $\operatorname{ccl}(N)$ contains exactly 8 elements. If $\tau=0, \mathfrak{C}(N F) \geqq\langle E\rangle\langle N F\rangle, \mathfrak{C}(N X F) \geqq\langle E X\rangle\langle N X F\rangle$; both centralizers have order $\geqq 16$. If $\tau=1, \mathfrak{C}(N F) \geqq\left\langle E^{2}, E F\right\rangle\langle N F\rangle, \mathbb{C}(N X F) \geqq\left\langle E^{2}, E F\right\rangle\langle N X F\rangle$ also have order $\geqq 16$. The classes of $N F, N X F$ therefore each contain exactly 4 elements.

\begin{tabular}{|l|l|l|l|l|}
\hline$N$ & $N F^{2 i}, N X F^{2 i}, 0 \leqq i \leqq 3$ & $\langle N, X, J\rangle$ & $2^{1+\rho}$ & $J^{\rho}$ \\
$N F$ & $N F, N F^{3}, N F^{5}, N F^{7}$ & $\langle E\rangle\langle N F\rangle$ if $\tau=0$, & $2^{1+\rho}$ & $J^{\rho}$ \\
& & $\left\langle E^{2}, E F\right\rangle\langle N F\rangle$ if $\tau=1$ & & \\
$N X F$ & $N X F, N X F^{3}, N X F^{5}, N X F^{7}$ & $\langle E X\rangle\langle N X F\rangle$ if $\tau=0$ & $2^{2-\rho}$ & $J^{1+\rho}$ \\
& & $\left\langle E^{2}, E F\right\rangle\langle N X F\rangle$ if $\tau=1$ & & \\
\hline
\end{tabular}


(c) Classes in $\mathfrak{P}-\mathfrak{N}$. Under repeated transformations by $F$, we have $E \sim E X \sim E J \sim E X J, E F^{2} \sim E X F^{2} \sim E X F^{-2} \sim E F^{-2}, E F \sim E X F \sim E X F^{5} \sim E F^{5}$, $E F^{3} \sim E X F^{3} \sim E X F^{-1} \sim E F^{-1}$. If $\tau=0$ we may use the results on $\mathscr{C}(N F)$ and the fact that $E X F^{2}=E^{3}$ or $E^{-1}$ to conclude that $\mathfrak{C}(E)=\mathfrak{C}\left(E X F^{2}\right) \geqq\langle E\rangle\langle N F\rangle$, the latter being a subgroup of order 16. The classes $\operatorname{ccl}(E), \operatorname{ccl}\left(E X F^{2}\right)$ then each have exactly 4 elements. If $\tau=0, N: E F \rightarrow E X F^{-1} ; \operatorname{ccl}(E F)$ then has exactly 8 elements, since $\mathfrak{C}(E F) \geqq\langle E F, X, J\rangle$. If $\tau=1, N: E \rightarrow E X F^{2}$ and $\operatorname{ccl}(E)$ has exactly 8 elements. Note that $E$ is then a self-centralizing element of order 8 conugate to its odd powers. If $\tau=1$, then $\mathfrak{C}(E F) \geqq\langle E F\rangle\langle X, N F\rangle, \mathfrak{C}\left(E F^{3}\right)$ $\geqq\left\langle E F^{3}\right\rangle\left\langle X, N F^{-1}\right\rangle$, both centralizers then having order $\geqq 16$. The classes $\operatorname{ccl}(E F), \operatorname{ccl}\left(E F^{3}\right)$ each contain exactly 4 elements.

$\left.\begin{array}{|l|l|l|c|l|}E & E, E X, E X J, E J & 8 & E^{2} \\ E X F^{2} & E F^{2}, E X F^{2}, E X F^{-2}, E F^{-2} & \langle E\rangle\langle N\rangle\rangle & 8 & E^{-2} \\ E F & E F^{2 i+1}, E X F^{2 i+1}, 0 \leqq i \leqq 3 & \langle E F\rangle\langle X, J\rangle & 2^{2-\sigma} & J^{1-\sigma} \\ \hline E & E F^{2 i}, E X F^{2 i}, 0 \leqq i \leqq 3 & \langle E\rangle & 8 & E^{2} \\ E F & E F, E X F, E X F^{5}, E F^{5} & \langle E F\rangle\langle X, N F\rangle & 2^{2-\sigma} & J^{1-\sigma} \\ E F^{3} & E F^{3}, E X F^{3}, E X F^{-1}, E F^{-1} & \left\langle E F^{3}\right\rangle\left\langle X, N F^{-1}\right\rangle & 2^{2-\sigma} & J^{1-\sigma}\end{array}\right\} \tau=0$,

Under repeated transformations by $F$ and $X$, we have $E N \sim E N J \sim E N X F^{2}$ $\sim E N X F^{-2}, E N F^{2} \sim E N F^{-2} \sim E N X \sim E N X J$. If $\tau=0$, then $N: E N \rightarrow E N X$; since $E N$ has order $8, \operatorname{ccl}(E N)$ has exactly 8 elements. If $\tau=1$, then $\mathfrak{C}(E N) \geqq\langle E N\rangle\left\langle F^{2}, E F\right\rangle, \mathfrak{C}(E N X) \geqq\langle E N X\rangle\left\langle F^{2}, E F\right\rangle$; both centralizers have order $\geqq 16$, so that $\operatorname{ccl}(E N), \operatorname{ccl}(E N X)$ each contain exactly 4 elements. Finally, if $\tau=0, E N F, E N F^{-1}, E N F^{3}$ have order 8. Since $N: E N F \rightarrow E N X F^{-1}$, and $\mathscr{C}(E N F) \geqq\langle E, X, N F\rangle$ has order $\geqq 32, \operatorname{ccl}(E N F)$ has just 2 elements. Multiplication by $J$ yields another class $E N F^{5} \sim E N X F^{3}$. Transformation of $E N F^{3}$ by $E, N, F$ respectively yield $E N F^{-1}, E N X F^{-3}, E N X F$. Since $\mathbb{C}\left(E N F^{3}\right)$ $\geqq\left\langle E N F^{3}\right\rangle\langle X\rangle, \operatorname{ccl}\left(E N F^{3}\right)$ has exactly 4 elements. If $\tau=1$, transformation of $E N F$ by $E, N, F$ respectively give $E N F^{-1}, E N X F^{-3}, E N X F^{-1}$, and since $\mathfrak{C}(E N F) \geqq\left\langle\mathfrak{P}^{\prime}, E N F\right\rangle$, ccl $(E N F)$ has exactly 4 elements. Multiplication by yields the remaining class.

\begin{tabular}{|c|c|c|c|c|}
\hline $\begin{array}{l}E N \\
E N F \\
E N F^{-3} \\
E N F^{3}\end{array}$ & $\begin{array}{l}E N F^{2}, E N X F^{2}, 0 \leqq i \leqq 3 \\
E N F, E N X F^{-1} \\
E N F^{-3}, E N X F^{3} \\
E N F^{3}, E N F^{-1}, E N X F^{-3}, E N X F\end{array}$ & $\begin{array}{l}\langle E N\rangle \\
\langle E, X, N F\rangle \\
\langle E, X, N F\rangle \\
\left\langle E N F^{3}\right\rangle\langle X\rangle\end{array}$ & $\begin{array}{l}8 \\
8 \\
8 \\
8\end{array}$ & $\begin{array}{l}F \pm 2 \\
X F \pm 2 \\
X F \pm 2 \\
X F \pm 2\end{array}$ \\
\hline$E N$ & $E N, E N J, E N X F^{2}, E N X F^{-2}$ & $\langle E N\rangle\left\langle F^{2}, E F\right\rangle$ & $3+(-1)^{\rho+}$ & $J^{1+\rho+\sigma}$ \\
\hline$E N X$ & $E N X, E N X J, E N^{2} F, E N F^{-2}$ & $\langle E N X\rangle\left\langle F^{2}, E F\right\rangle$ & $3-(-1)^{\rho+}$ & $J^{\rho+\sigma}$ \\
\hline$E N F$ & $E N F, E N F^{-1}, E N X F^{-3}, E N X F^{-1}$ & $\left\langle\mathfrak{P}^{\prime}, E N F\right\rangle$ & 4 & $X J^{1+\rho+\sigma}$ \\
\hline$E N F^{3}$ & $E N F^{3}, E N F^{-3}, E N X F, E N X F^{3}$ & $\left\langle\mathfrak{P}^{\prime}, E N F\right\rangle$ & 4 & $X J^{\rho+\sigma}$ \\
\hline
\end{tabular}


2. Let $\mathfrak{P}=\mathfrak{P}(\rho, \sigma, \tau)$ be the group of order 64 considered in $\$ 1$. We assume in this section that $\mathfrak{P}$ is a Sylow subgroup of a larger group $\mathfrak{b}$. Let $\mathfrak{B}^{*}$ be the focal subgroup of $\mathfrak{P}$ in $\mathfrak{G}$, i.e. $\mathfrak{P}^{\#}\left\langle Q P^{-1}\right| Q=P \in \mathfrak{P}$ and $Q \sim P$ in $\left.\mathfrak{G}\right\rangle$. The $S_{2}$-subgroup of $\mathfrak{5} / \mathfrak{G}^{\prime}$ is then isomorphic to $\mathfrak{P} / \mathfrak{B}^{\#}$ by [1], Theorem 8 .

LeMma (2A). $E^{2} \sim F^{2}$ in $(5$.

Proof. Suppose $E^{2} \sim F^{2}$ in $\mathfrak{5}$. Since $\left\langle E^{2}\right\rangle$ and $\left\langle F^{2}\right\rangle$ are normal in $\mathfrak{P}$, there exists by Burnside's theorem [12, Lemma, p. 139] a $G \in \mathfrak{N}(\mathfrak{P})$ such that $G:\left\langle E^{2}\right\rangle \rightarrow\left\langle F^{2}\right\rangle$; since $F^{2} \sim F^{-2}$ in $\mathfrak{P}$ we may assume that $G: E^{2} \rightarrow F^{2}$, and that $G$ has odd order. $G$ then permutes the four self-centralizing cyclic subgroups of $\mathfrak{P}$ of order $8,\langle F\rangle,\langle X F\rangle,\langle E N\rangle,\langle E N X\rangle$ if $\tau=0 ;\langle F\rangle,\langle X F\rangle,\langle E\rangle,\left\langle E F^{2}\right\rangle$ if $\tau=1$. Hence $G$ fixes one of these subgroups, and then $G$ would fix $E^{2}$ or $F^{2}$, which is impossible. Thus $E^{2} \sim F^{2}$ in 5 .

Lemma (2B). Suppose $Y \sim Z$, where $Y, Z$ are elements of order 2 or 4 in $\mathfrak{P}$, and $v c_{\mathfrak{P}}(Y)=3, v c_{\mathfrak{P}}(Z)=5$. Then $\operatorname{ccl}(J)$ is not isolated.

Proof. The assumptions imply that $Y \in \operatorname{ccl}(N)$ or $\operatorname{ccl}(E F)$ if $\tau=0, Y \in \operatorname{ccl}(N)$ if $\tau=1$. Suppose $\operatorname{ccl}(J)$ is isolated. Let $\mathfrak{C}^{\circ}(Y)=\langle T \in \mathbb{C}(J)| T: Y \rightarrow Y$ or $\left.Y J\right\rangle$, and let $\mathfrak{L}=\mathbb{C}_{\mathfrak{P}}^{0}(Y)=\mathfrak{C}^{0}(Y) \cap \mathfrak{P} . \quad \mathfrak{L}=\left\langle\mathfrak{P}^{\prime}, N\right\rangle$ or $\left\langle\mathfrak{P}^{\prime}, E F\right\rangle$ according as $Y=N$ or $Y=E F$; thus $\mathbb{L} \leqslant \mathfrak{P}$. Since $\operatorname{ccl}(J)$ is isolated, $Y \sim Z$ in $\mathbb{C}(J)$. In particular, there exists $G \in \mathbb{C}(J)$ such that $G: Y \rightarrow Z$, and we may assume $G: \mathbb{C}^{\circ}(Y) \rightarrow \mathbb{C}^{\circ}(Z)$. Let $\mathfrak{P}_{1}$ be an $S_{2}$-subgroup of $\mathfrak{C}^{\circ}(Y)$ containing $\mathfrak{L} ; \mathfrak{P}_{1}$ is then a Sylow subgroup of $\mathfrak{G}$. If $\mathfrak{Q} \unlhd \mathfrak{P}_{1}$, then there exists an $H \in \mathfrak{N}(\mathfrak{L})$ such that $H: \mathfrak{P}_{1} \rightarrow \mathfrak{P}$. If $Z_{1}$ is the image of $Y$ under $H$, then necessarily $v c_{\mathfrak{p}}\left(Z_{1}\right)=5$ and $Z_{1} \in \mathfrak{P}^{\prime}$. In particular, the images of $E^{2}, F^{2}$ under $H$ are elements of $\mathfrak{Q}$ not commuting with $Z_{1}$; in other words, they lie in $\mathfrak{L}-\mathfrak{P}^{\prime}=\operatorname{ccl}(Y)$. But then $E^{2} \sim F^{2}$, which is impossible by (2A). Hence $\mathfrak{R}$ is not a normal subgroup of $\mathfrak{P}_{1}$. Choose $\mathfrak{M}$ such that $\mathfrak{L} \triangleleft \mathfrak{M} \triangleleft \mathfrak{P}_{1}$, and an $S_{2}$-subgroup $\mathfrak{P}_{2}$ of $\mathfrak{G}$ such that $\mathfrak{B}<\mathfrak{P}_{2} \leqq \mathfrak{N}(\mathfrak{L})$. Then there exists an $H \in \mathfrak{N}(\mathfrak{L})$ such that $H: \mathfrak{P} \rightarrow \mathfrak{P}_{2}$. Since $H$ must fix $J$, there are elements $Y_{1}, Y_{2}$ in $\operatorname{ccl}(Y)$ such that $H: Y_{1} \rightarrow Y_{2}$. This implies $v c_{\mathfrak{B}_{2}}(Y)=3$. However, $v c_{\mathfrak{B}_{2}}(Y) \geqq 4$, since $v c_{\mathfrak{P}_{1}}(Y)=5$. Thus $\operatorname{ccl}(J)$ is not isolated.

Proposition (2C). If $\tau=0$, then $\mathfrak{P}^{\#}<\mathfrak{P}$ :and $\mathfrak{G}$ has a subgroup of index 2 .

Proof. From the tables in $\S 1, J$ is the only involution in $\mathfrak{P}$ which is a square in $\mathfrak{P}$. Suppose $G: Y \rightarrow J$ for some $Y \neq J$ in $\mathfrak{P}$ and some $G$ in $\mathfrak{G}$. We may assume $G: \mathfrak{C}_{\mathfrak{p}}(Y) \rightarrow \mathfrak{P}$. Let $\mathfrak{P}_{1}$ be an $S_{2}$-subgroup of $\mathfrak{C}(Y)$ containing $\mathfrak{C}_{\mathfrak{P}}(Y)$. For every $T \in \mathfrak{P}_{1}, T^{4} \in\langle Y\rangle$. If $v c_{\mathfrak{P}}(Y) \geqq 4$, we may assume $Y=X, N F$, or $N X F$; then $E N F \in \mathfrak{C}_{\mathfrak{B}}(Y)$. But this is impossible, since $(E N F)^{4}=J$. If $v \mathcal{C}_{\mathfrak{P}}(Y)=3$, we may assume $Y=N$ or $E F$. If $Y=N$, then $G$ must transform $N X$ into $\operatorname{ccl}(N)$ or $\operatorname{ccl}(E F)$ by what has just been proved. If $G: N X \rightarrow \operatorname{ccl}(N)$, then $G: X=N(N X) \rightarrow$ $J \operatorname{ccl}(N)=\operatorname{ccl}(N)$, which is impossible. If $G: N X \rightarrow \operatorname{ccl}(E F)$, then $G: X \rightarrow J \operatorname{ccl}(E F)$ $\stackrel{0}{=} \operatorname{ccl}(E F)$, which is also impossible. If $Y=E F$, a similar argument can be applied to $E F X$. Thus $\operatorname{ccl}(J)$ is isolated. 
Let $Y=N$ or $E F$, and suppose $Y \sim N F$ or $Y \sim N X F$. Then there exists a $G \in \mathbb{G}$ with $G: Y \rightarrow N F$ or $N X F$, and $G: \mathfrak{C}_{\mathfrak{P}}(Y) \rightarrow \mathfrak{C}_{\mathfrak{P}}(N F)$ or $\mathfrak{C}_{\mathfrak{B}}(N X F)$ by (2B). Now $X \in \mathfrak{C}_{\mathfrak{B}}(Y)$; therefore $G: X \rightarrow \operatorname{ccl}(N F)$ or $\operatorname{ccl}(N X F)$. But this is impossible by (2B), since it would imply $Y \sim X$. Hence the only possible fusions among elements of order 2 and 4 are between $X, N F$ or $N X F|N, E F| E^{2}, N F$ or $N X F \mid F^{2}, N F$ or $N X F$. By (2A) the only possible fusions among elements of order 8 are between $F, E N \mid E, E F^{2}, E N F, E N F^{-3}, E N F^{3}$. These fusions, if they occur, contribute at most $\left\langle\mathfrak{P}^{\prime}, E, N F\right\rangle$ to $\mathfrak{P}^{\#}$, and thus $\mathfrak{P}^{\#}<\mathfrak{P}$.

From now on we assume $\tau=1$, since we want to find out which $\mathfrak{P}$ can occur as an $S_{2}$-subgroup of a group without subgroups of index 2 .

Lemma (2D). Suppose $\tau=1$ and $\operatorname{ccl}(J)$ is not isolated. Then $\rho=0, \sigma=1$. Moreover $X \sim J$, and after a suitable relabeling of the generators $E, N, F$, we may assume $E^{2} \sim E N F^{3}, F^{2} \sim E N F, N \sim E F$.

Proof. There exists $Y \neq J$ in $\mathfrak{P}$ such that $Y \sim J$ in $(5)$ We first show we may take $Y=X$. Choose $G \in \mathbb{F}$ such that $G: Y \rightarrow J$ and $G: \mathfrak{C}_{\mathfrak{P}}(Y) \rightarrow \mathfrak{P}$. If $v c_{\mathfrak{P}}(Y)=4$, then $J$ is a square in $\mathfrak{C}_{\mathfrak{P}}(Y)$. Its image under $G$ is an involution $\neq J$ which is a square in $\mathfrak{P}$. Hence $G: J \rightarrow \operatorname{ccl}(X)$ and $X \sim J$. If $Y=N$, and if $G: N X \rightarrow \operatorname{ccl}(X)$ or $\operatorname{ccl}\left(Y_{1}\right)$ with $v c_{\Re}\left(Y_{1}\right)=4$, then $X \sim J$. But if $G: N X \rightarrow \operatorname{ccl}(N)$, then $G: X=N(N X) \rightarrow J \operatorname{ccl}(N)=\operatorname{ccl}(N)$. Hence $X \sim J$ in all cases.

Let $\mathfrak{U}=\mathfrak{C}_{\mathfrak{P}}(X)=\left\langle\mathfrak{P}^{\prime}, E F, N\right\rangle$, and let $\mathfrak{P}_{1}$ be an $S_{2}$-subgroup of $\mathfrak{C}(X)$ containing $\mathfrak{U}$. Since $\mathfrak{U} \triangleleft \mathfrak{P}, \mathfrak{U} \triangleleft \mathfrak{P}_{1}$, there exists $B \in \mathfrak{N}(\mathfrak{U})$ such that $B: \mathfrak{P} \rightarrow \mathfrak{P}_{1}$, $B: J \rightarrow X$. Furthermore, $B$ necessarily maps $X \rightarrow X J$, and hence $B: X J \rightarrow J$. We may therefore assume $B$ has order a power of 3 , if we only use the properties that $B \in \mathfrak{N}(\mathfrak{U})$, and $B: J \rightarrow X \rightarrow X J \rightarrow J$ in the remainder of this proof. If $\rho=1$, the 8 elements of $\operatorname{ccl}(N) \subseteq \mathfrak{U}$ are mapped by $B$ onto 8 elements of $\mathfrak{U}$ whose squares are $X$. Since there are only 4 such elements in $\mathfrak{U}, \rho$ must be 0 . Similarly, to show $\sigma=1$, the same argument can be applied to the 8 elements of $\operatorname{ccl}(E F) \cup \operatorname{ccl}\left(E F^{3}\right)$. $B$ maps $E^{2}, F^{2}$ onto elements of $\mathfrak{U}$ whose squares are $X$. Since the map $E \rightarrow F$, $F \rightarrow E, N \rightarrow N$ defines an automorphism of $\mathfrak{P}$, we may assume $F^{2} \sim E N F$, $E^{2} \sim E N F^{3}$. Indeed, if $E^{2} \sim E N F$, then in the new notation $F^{2} \sim F N E \sim E F N$ $\sim E N F$. Finally, $B: \operatorname{ccl}(N) \rightarrow \operatorname{ccl}(N) \cup \operatorname{ccl}(E F) \cup \operatorname{ccl}\left(E F^{3}\right)$, the latter being a characteristic set of $\mathfrak{U}$. Suppose $B: \operatorname{ccl}(N) \rightarrow \operatorname{ccl}(N)$. Since $B$ has order a power of 3 , and $\operatorname{ccl}(N)$ has 8 elements, it follows that $B$ fixes at least two elements $N_{1} \neq N_{2}$ of $\operatorname{ccl}(N)$. But then $B$ would fix $N_{1} N_{2}^{-1} \in \mathfrak{P}^{\prime}$, which is impossible. Hence $N \sim E F^{\alpha}$ for some $\alpha=1$ or 3 . The map $E \rightarrow F^{3}, F \rightarrow E, N \rightarrow N$ defines an automorphism of $\mathfrak{P}$ which interchanges $\operatorname{ccl}(E), \operatorname{ccl}(F)$, as well as $\operatorname{ccl}(E N F), \operatorname{ccl}\left(E N F^{3}\right)$, so that we may assume $\alpha=1$.

COROllaRY (2E). Under the assumptions of (2D) there is a BE(5 of order a power of 3 such that

$$
B: J \rightarrow X \rightarrow X J \rightarrow J
$$


Moreover, B either fuses $\operatorname{ccl}(N), \operatorname{ccl}(E F), \operatorname{ccl}\left(E F^{3}\right)$ or $B$ fuses $\operatorname{ccl}(N), \operatorname{ccl}(E F)$ and $B: \operatorname{ccl}\left(E F^{3}\right) \rightarrow \operatorname{ccl}\left(E F^{3}\right)$. In either case $B$ maps the union of these three classes onto itself.

LemMa (2F). Suppose $\tau=1$. Let $Y$ be one of the elements $E N, N F, E N X, N X F$, $E F, E F^{3} ;$ and $Z$ one of the elements $X, E^{2}, F^{2}$. Let $\mathfrak{L}=\left\langle\mathfrak{P}^{\prime}, E N, N F\right\rangle$. If $Y \sim Z$ in $\mathfrak{G}$, then there exists $a G \in \mathfrak{G}$ such that $G: J \rightarrow J, Y \rightarrow Z$, and $\mathfrak{C}_{\mathfrak{p}}(Y) \rightarrow \mathfrak{L} \cap \mathfrak{C}_{\mathfrak{B}}(Z)$.

Proof. We first show that $Y \sim Z$ in $\mathbb{C}(J)$. If $Y, Z$ have order 4 , then $Y^{2}=Z^{2}=J$, and this is clear. Suppose then that $Z=X$. Let $\mathfrak{P}_{1}$ be an $S_{2}$-subgroup of $\mathfrak{C}(X)$ such that $\mathfrak{P}_{1} \geqq \mathfrak{C}_{\mathfrak{B}}(X)$. Then $X, X J, J$ are the only involutions in $\mathfrak{P}_{1}$ which are squares of elements in $\mathfrak{P}_{1}$, since they are already squares of elements in $\mathbb{C}_{\mathfrak{p}}(X)$. Now there exists $A \in \mathfrak{G}$ such that $A: \mathfrak{C}_{\mathfrak{P}}(Y) \rightarrow \mathfrak{P}_{1}$, and $A: Y \rightarrow X$. Since $J$ is a square in $\mathfrak{C}_{\mathfrak{p}}(Y), A: J \rightarrow J$ or $X J$. If $A: J \rightarrow J$, then $Y \sim X$ in $\mathfrak{C}(J)$. If $A: J \rightarrow X J$, then $\operatorname{ccl}(J)$ is not isolated. If $B$ is the element of (2E), then $A B: J \rightarrow J, Y \rightarrow X J$, and $Y \sim X$ in $\mathbb{C}(J)$. Thus in all cases, $Y \sim Z$ in $\mathbb{C}(J)$.

Let $\mathbb{C}^{0}(Y)=\langle T \in \mathbb{C}(J)| T: Y \rightarrow Y$ or $\left.Y J\right\rangle . \mathfrak{L}$ is then $\mathbb{C}^{0}(Y) \cap \mathfrak{P}$ and $\mathfrak{L} \triangleleft \mathfrak{P}$. Let $\mathfrak{P}_{1}$ be an $S_{2}$-subgroup of $\mathfrak{C}(Y)$ containing $\mathfrak{L}$. By the above, $\left(\mathfrak{P}_{1}: \mathfrak{Q}\right)=2$ and $\mathfrak{L} \triangleleft \mathfrak{P}_{1}$. Let $G \in \mathfrak{N}(\mathfrak{L})$ such that $G: \mathfrak{P}_{1} \rightarrow \mathfrak{P} . G$ fixes $J$, since $J$ is the only involution in $\mathfrak{L}$ which is a square in $\mathfrak{L}$. The image of $Y$ under $G$ has a centralizer in $\mathfrak{P}$ of order 32, and hence belongs to one of the classes $\operatorname{ccl}(X), \operatorname{ccl}\left(E^{2}\right), \operatorname{ccl}\left(F^{2}\right)$. Since no two of these classes can be fused in $\mathfrak{G}$, we may assume $G: Y \rightarrow Z$. In particular, $G: \mathfrak{C}_{\mathfrak{P}}(Y) \rightarrow \mathfrak{C}_{\mathfrak{B}}(Z) \cap \mathfrak{Q}$.

In the table below the entry in the $T_{i}$-row and $T_{j}$-column is the number of elements in $\mathfrak{C}_{\mathfrak{p}}\left(T_{i}\right) \cap \operatorname{ccl}\left(T_{j}\right)$. The union of the classes indexing the columns is $\mathfrak{R}$.

\begin{tabular}{l|ccccccc|cc|cc|}
\multicolumn{1}{c}{} & 1 & $J$ & $X$ & $N F$ & $N X F$ & $E N$ & $E N X$ & $E F$ & $E F^{3}$ & $E^{2}$ & $F^{2}$ \\
$N F$ & 1 & 1 & 0 & 2 & 2 & 4 & 0 & 2 & 2 & 2 & 0 \\
$N X F$ & 1 & 1 & 0 & 2 & 2 & 0 & 4 & 2 & 2 & 2 & 0 \\
$E N$ & 1 & 1 & 0 & 4 & 0 & 2 & 2 & 2 & 2 & 0 & 2 \\
$E N X$ & 1 & 1 & 0 & 0 & 4 & 2 & 2 & 2 & 2 & 0 & 2 \\
$E F$ & 1 & 1 & 2 & 2 & 2 & 2 & 2 & 4 & 0 & 0 & 0 \\
$E F^{3}$ & 1 & 1 & 2 & 2 & 2 & 2 & 2 & 0 & 4 & 0 & 0 \\
\cline { 2 - 11 } & 1 & 1 & 2 & 0 & 0 & 0 & 0 & 4 & 4 & 2 & 2 \\
$E^{2}$ & 1 & 1 & 2 & 4 & 4 & 0 & 0 & 0 & 0 & 2 & 2 \\
$F^{2}$ & 1 & 1 & 2 & 0 & 0 & 4 & 4 & 0 & 0 & 2 & 2 \\
\hline
\end{tabular}

Proposition (2G). Suppose $\tau=1$. If $\operatorname{ccl}(J)$ is isolated in $\mathfrak{F}$, then $\mathfrak{P}^{*}<\mathfrak{P}$.

Proof. (a) Suppose $\operatorname{ccl}(N)$ is isolated. The only possible fusions are between $E N F, E N F^{3} \mid X, E^{2}, F^{2}, N F, N X F, E N, E N X, E F, E F^{3}$. In this case

$$
\mathfrak{P}^{*} \leqq\left\langle\mathfrak{P}^{\prime}, E N, N F\right\rangle\langle\mathfrak{P},
$$

and we are done. Thus we may assume $\operatorname{ccl}(N)$ is not isolated. 
(b) Suppose $\operatorname{ccl}(X)$ is isolated. Let $Y$ be one of $E N, N F, E N X, N X F$. If $G: N \rightarrow Y$ we may assume $G: \mathfrak{C}_{\mathfrak{P}}(N) \rightarrow \mathfrak{C}_{\mathfrak{\beta}}(Y)$. In particular the image of $X$ under $G$ is not in $\operatorname{ccl}(X)$, since $\mathfrak{C}_{\Re}(Y) \cap \operatorname{ccl}(X)=\varnothing$, and this is impossible. By $(2 F)$, (2.1) $Y \nsim E^{2}, Y \nsim F^{2}$, since either fusion implies that $\operatorname{ccl}(X)$ is not isolated. Hence $v c(Y)=4$; by $(2 F),(2.1)$ it also follows that $Y \sim E F, Y \sim E F^{3}$. (The results of $(2 \mathrm{~F})$ and $(2.1)$ are valid in case $v c(Y)=4$.) Also $N \sim E^{2}, N \sim F^{2}$ by (2B). The only possible fusions in $\left(5\right.$ are between $N, E F, E F^{3}|N F, N X F, E N, E N X| E N F, E N F^{3}$. But then $\mathfrak{P}^{\#} \leqq\left\langle\mathfrak{P}^{\prime}, E N F, N, E F\right\rangle<\mathfrak{P}$ and we are done. Thus we may assume $\operatorname{ccl}(X)$ is not isolated.

(c) Suppose $\sigma=0$. Then $N, N F, E N X$ have order $2^{\rho+1} ; E N, N X F$ have order $2^{2-\rho} ; E F, E F^{3}$ have order 4 . By (a) and (2B), $N \sim Y$ for some $Y \in \mathfrak{P}$ with $v c_{\mathfrak{P}}(Y)=v c(Y)=4$. Let $G \in \mathbb{G}$ such that $G: N \rightarrow Y$ and $G: \mathfrak{C}_{\mathfrak{P}}(N) \rightarrow \mathfrak{C}_{\mathfrak{P}}(Y)$. Now $\mathfrak{C}_{\mathfrak{B}}(N) \cap \operatorname{ccl}(N)=\{N, N J, N X, N X J\}$; but $\mathfrak{C}_{\mathfrak{B}}(Y) \cap(\operatorname{ccl}(N F) \cup \operatorname{ccl}(E N X))$ has only 2 elements by (2.1). Hence $G$ necessarily fuses $\operatorname{ccl}(N)$ with $\operatorname{ccl}\left(E F^{\alpha}\right)$ for some $\alpha=1$ or 3 . In other words, we may assume $Y=E F^{\alpha}$. In particular, $\rho=1$, and $N, N F, E N X$ have order 4. If $\mathbb{C}_{\mathfrak{P}}^{0}(N)=\langle T \in \mathfrak{P}| T: N \rightarrow N$ or $\left.N J\right\rangle$, we may further assume $G: \mathbb{C}_{\mathfrak{P}}^{0}(N) \rightarrow \mathfrak{C}_{\mathfrak{\beta}}^{0}\left(E F^{\alpha}\right)=\mathfrak{L}, \mathfrak{L}$ as in $(2 \mathrm{~F})$. By $(2 \mathrm{~F}),(2.1)$ the 4 elements of $\operatorname{ccl}(N)$ in $\mathfrak{C}_{\mathfrak{P}}^{0}(N)-\mathfrak{C}_{\mathfrak{p}}(N)$ are mapped by $G$ into

$$
\left(\operatorname{ccl}\left(E F^{\beta}\right) \cup \operatorname{ccl}(N F) \cup \operatorname{ccl}(E N X)\right) \cap\left(\mathfrak{C}_{\mathfrak{p}}^{0}\left(E F^{\alpha}\right)-\mathfrak{C}_{\mathfrak{P}}\left(E F^{\alpha}\right)\right),
$$

where $\beta=1$ or $3, \beta \neq \alpha$. Thus $N \sim E F \sim E F^{3}$ or $N \sim E F^{\alpha} \sim N F \sim E N X$ by (2.1). Suppose we have the latter case. Then $E F^{\alpha} \sim N F$ implies by (2F), (2.1) that $E^{2}$ is fused to one of $N F, E N X$ or $E F^{\alpha}$ and hence $N \sim E^{2}$, which is impossible by (2B). Suppose we have the first case $N \sim E F \sim E F^{3}$. By (b) $\operatorname{ccl}(X)$ is not isolated and hence $X \sim E N$ or $X \sim N X F$. If $X \sim E N$, then by (2F), (2.1) and (2A), $N F \sim E F \sim E F^{3} \sim N$, and necessarily $E N X \sim E^{2}$. The fusions introduced are $X \sim E N, N \sim E F \sim E F^{3} \sim N F, E^{2} \sim E N X$. Similarly, if $X \sim N X F$, then $X \sim N X F, N \sim E F \sim E F^{3} \sim E N X, F^{2} \sim N F$. Thus $X \sim E N$ or $X \sim N X F$, but not both. The only other possible fusions are $E N F \sim E N F^{3}$. In all cases, $\mathfrak{P}^{\#}$ $\leqq\left\langle\mathfrak{P}^{\prime}, E N, F\right\rangle$ or $\mathfrak{P}^{\#} \leqq\left\langle\mathfrak{P}^{\prime}, N F, E\right\rangle$, and so $\mathfrak{P}^{\#}<\mathfrak{P}$.

(d) Suppose $\sigma=1$. Then $N, E N, N F$ have order $2^{\rho+1} ; N X F, E N X$ have order $2^{2-\rho} ; E F, E F^{3}$ have order 2. By (a) and (2B), $N \sim Y$ for some $Y \in \mathfrak{P}$ with $v c_{\mathfrak{P}}(Y)=v c(Y)=4$. We may assume $Y=E N, N F$, or $E F^{\alpha}$ for some $\alpha=1$ or 3. Let $G \in \mathfrak{G}$ such that $G: N \rightarrow Y$ and $\mathfrak{C}_{\mathfrak{P}}(N) \rightarrow \mathfrak{C}_{\mathfrak{B}}(Y)$. If $\mathfrak{C}_{\mathfrak{P}}^{0}(N)=\langle T \in \mathfrak{P}| T: N \rightarrow N$ or $\left.N J\right\rangle$, we may further assume $G: \mathfrak{C}_{\mathfrak{P}}^{0}(N) \rightarrow \mathfrak{C}_{\mathfrak{P}}^{0}(Y)=\mathfrak{L}$, $\mathfrak{L}$ as in $(2 \mathrm{~F})$. The 4 elements of $\operatorname{ccl}(N)$ in $\mathfrak{C}_{\mathfrak{P}}^{0}(N)-\mathfrak{C}_{\mathfrak{P}}(N)$ are mapped into $\mathfrak{L}-\mathfrak{C}_{\mathfrak{P}}(Y)$; hence if $Y=E N$ or $N F$, then $N \sim E F^{\alpha}$. Therefore $N \sim E F^{\alpha}$ for some $\alpha=1$ or 3 and $\rho=0$. In particular, it follows that $E^{2} \sim N X F, F^{2} \sim E N X, E N X \approx N X F$. Indeed, if any of these were not the case, then by (2F), l(2.1) $E^{2} \sim F^{2}$ which is impossible by (2A). By (b) $\operatorname{ccl}(X)$ is not isolated. If $X \sim N F$, then $F^{2} \sim N X F$, $E N \sim E F \sim E F^{3}$ by $(2 \mathrm{~F}),(2.1)$. The only possible fusions in $(\mathfrak{G}$ are $X \sim N F$, $E N \sim E F \sim E F^{3} \sim N, E N F \sim E N F^{3}, F^{2} \sim N X F, E^{2} \sim E N X$. If $E^{2} \sim E N X$ 
occurred, then by (2F), (2.1) $N F$ would be fused to one of $E N, E F, E F^{3}$, and hence $X \sim N$, which is impossible by (2B). Computing $\mathfrak{P}^{\#}$, we have $\mathfrak{P}^{\#} \leqq\left\langle\mathfrak{P}^{\prime}, E, N F\right\rangle\langle\mathfrak{P}$, and we are done. If $X \sim E N$, then by a similar argument, the only possible fusions are $X \sim E N, N F \sim E F \sim E F^{3} \sim N, E N F \sim E N F^{3}$ $E^{2} \sim E N X$, and $\mathfrak{P}^{\#} \leqq\left\langle\mathfrak{P}^{\prime}, E N, F\right\rangle\left\langle\mathfrak{P}\right.$. We may finally suppose $X \sim E F^{\beta}$, $\beta=1$ or $3, \beta \neq \alpha$. By $(2 \mathrm{~F}),(2.1)$ we then have $E^{2} \sim E N X, F^{2} \sim N X F, N F$ $\sim E N \sim E F^{\alpha} \sim N, X \sim E F^{\beta}$.

(e) Since $N \sim N F^{2} \sim E N$, there exist $G_{1}, G_{2} \in \mathbb{G}$ such that $G_{1}: N \rightarrow E N$, $\mathfrak{C}_{\mathfrak{P}}(N) \rightarrow \mathfrak{C}_{\mathfrak{P}}(E N)$ and $G_{2}: N F^{2} \rightarrow E N, \mathfrak{C}_{\mathfrak{P}}\left(N F^{2}\right) \rightarrow \mathfrak{C}_{\mathfrak{P}}(E N)$. In particular, $G_{1}$ and $G_{2}$ both map $X$ into $\operatorname{ccl}\left(E F^{\beta}\right) \cap \mathfrak{C}_{\mathfrak{p}}(E N)$. Conjugating if necessary by an element in $\mathfrak{C}_{\mathfrak{P}}(E N)$, we may assume both $G_{1}$ and $G_{2}$ map $X$ onto $E F^{\beta}$. Set $G=G_{1} G_{2}^{-1}$; then $G: J \rightarrow J, X \rightarrow X, N \rightarrow N F^{2}$. If $\mathfrak{A}=\langle X, J\rangle, \mathfrak{H}=\mathfrak{C}(\mathfrak{A})$, then $\left\langle\mathfrak{A}, F^{2}, E F, N\right\rangle$ is an $S_{2}$-subgroup of $\mathfrak{H}$. In particular, the $S_{2}$-subgroups of $\mathfrak{S} / \mathfrak{A}$ are elementary abelian of order 8 . Modulo $\mathfrak{A}$ the following elements represent the elements of an $S_{2}$-subgroup of $\mathfrak{H} / \mathfrak{A}: 1 F^{2} E F N E F^{3} N F^{2} E N F^{-1} E N F$. Moreover, $N \sim N F^{2}$ in $\mathfrak{Y} / \mathfrak{A}$ by the above. By Burnside's theorem we see that either all the nonidentity representatives above are fused in $\mathfrak{H} / \mathfrak{A}$, or 6 of them are fused in sets of 3 each. But this is impossible, since 5 of them have squares in $\langle J\rangle$ and 2 of them have squares in $\operatorname{ccl}(X)$. This completes the proof.

REMARK. We note that if $\mathfrak{P}=\mathfrak{P}(\rho, \sigma, \tau)$ with $\rho=0, \sigma=\tau=1$, and if $\mathfrak{U}=\langle J, X\rangle$, then as a consequence of $(\mathrm{e})$, the group $\mathfrak{C}(\mathfrak{U})$ has a normal 2-complement.

Proposition (2H). Let $\rho=0, \sigma=1, \tau=1$. If $(5$ has no subgroups of index 2 , then after a suitable relabeling of the generators $E, N, F$, there are exactly three possibilities for the fusion of involutions of $\mathfrak{P}$.

I. $J \sim X \sim N \sim E F \sim E F^{3} \sim N F \sim E N$,

II. $J \sim X \sim N \sim E F, E F^{3} \sim N F \sim E N$,

III. $J \sim X \sim E F^{3}, N \sim E F \sim N F \sim E N$.

In all three cases, $E^{2} \sim E N X \sim E N F^{3}, F^{2} \sim N X F \sim E N F$.

Proof. The following are consequences of (2D) and (2F), (2.1): $E^{2} \sim N X F, F^{2} \approx E N X$, since either fusion implies $E^{2} \sim F^{2}$. Secondly, if $X \sim E F^{\alpha}$ for some $\alpha=1$ or 3 , then $E^{2} \sim E N X, F^{2} \sim N X F, N F \sim E N \sim E F^{\beta}$, where $\beta=1$ or $3, \beta \neq \alpha$. By $(2 \mathrm{G})$ and $(2 \mathrm{E}), J \sim X, N \sim E F, E^{2} \sim E N F^{3}$, $F^{2} \sim E N F$. Suppose $N \sim X$. Then $E F \sim X$, and we have either cases I or II. Suppose that neither case I nor case II holds. If $X \sim J$ is fused to no other class, then the only possible fusions in $(5$ are between

$$
X \sim J\left|E^{2} \sim E N F^{3}\right| F^{2} \sim E N F\left|N \sim E F, E F^{3}\right| N F, E N
$$

by $(2 \mathrm{~F}),(2.1)$. But then $\mathfrak{P}^{\#} \leqq\left\langle\mathfrak{P}^{\prime}, E N F, E F\right\rangle\langle\mathfrak{P}$, which is impossible. Thus $X \sim Y$ for some $Y \in \mathfrak{P}$ with $v c_{\mathfrak{P}}(Y)=4$. If $Y=E F^{3}$, then we have case III by an 
above remark. If $Y=N F$, then $E N \sim E F \sim E F^{3} \sim N$ by (2F), (2.1). In order that $\mathfrak{P}^{\#}=\mathfrak{P}, E^{2}$ must be fused to $E N X$. But then $E F \sim X$ by (2F), (2.1) and $N \sim X$, which has been excluded. Similarly, if $Y=E N$, a contradiction is also obtained. This completes the proof.

Summarizing the results of $\S \S 1,2$, we have:

TheOREM (2I). Let $\mathfrak{G}$ be a finite group of order $64 m, m$ odd. Let $\mathfrak{P}$ be an $S_{2}$-subgroup of $(\mathfrak{b}$. Suppose there is an element $F \in \mathfrak{P}$ of order 8 which is selfcentralizing in $\mathfrak{P}$ and conjugate to its odd powers in $\mathfrak{P}$. If $\mathbb{5}$ has no subgroups of index 2 , then $\mathfrak{P}$ is isomorphic to $\mathfrak{P}(\rho, \sigma, \tau)$ of $(1 \mathrm{~A})$, with $\rho=0, \sigma=\tau=1$. Furthermore, if notation is chosen suitably, the possibilities for the fusion of the 2-power classes of $\mathfrak{6}$ are

I. $F|E| E^{2} \sim E N X \sim E N F^{3}\left|F^{2} \sim N X F \sim E N F\right| J \sim X \sim N \sim E F \sim E F^{3} \sim N F \sim E N$,

II. $F|E| E^{2} \sim E N X \sim E N F^{3}\left|F^{2} \sim N X F \sim E N F\right| J \sim X \sim N \sim E F \mid E F^{3} \sim N F \sim E N$,

III. $F|E| E^{2} \sim E N X \sim E N F^{3}\left|F^{2} \sim N X F \sim E N F\right| J \sim X \sim E F^{3} \mid N \sim E F \sim N F \sim E N$.

Let $\mathfrak{P}$ be the 2-group of (2I). The character table of $\mathfrak{P}$ is easily seen to be

\begin{tabular}{rrrrrrrrrrrrrrrrrr}
$E$ & $F$ & $F^{2}$ & $N X F$ & $E N F$ & $E^{2}$ & $E N X$ & $E N F^{3}$ & $E N$ & $N F$ & $E F^{3}$ & $E F$ & $N$ & $X$ & $J$ & 1 \\
\hline 1 & 1 & 1 & 1 & 1 & 1 & 1 & 1 & 1 & 1 & 1 & 1 & 1 & 1 & 1 & 1 \\
1 & 1 & 1 & -1 & -1 & 1 & -1 & -1 & -1 & -1 & 1 & 1 & -1 & 1 & 1 & 1 \\
1 & -1 & 1 & -1 & -1 & 1 & 1 & -1 & 1 & -1 & -1 & -1 & 1 & 1 & 1 & 1 \\
1 & -1 & 1 & 1 & 1 & 1 & -1 & 1 & -1 & 1 & -1 & -1 & -1 & 1 & 1 & 1 \\
-1 & 1 & 1 & 1 & -1 & 1 & -1 & -1 & -1 & 1 & -1 & -1 & 1 & 1 & 1 & 1 \\
-1 & 1 & 1 & -1 & 1 & 1 & 1 & 1 & 1 & -1 & -1 & -1 & -1 & 1 & 1 & 1 \\
-1 & -1 & 1 & -1 & 1 & 1 & -1 & 1 & -1 & -1 & 1 & 1 & 1 & 1 & 1 & 1 \\
-1 & -1 & 1 & 1 & -1 & 1 & 1 & -1 & 1 & 1 & 1 & 1 & -1 & 1 & 1 & 1 \\
\hline 0 & 0 & 2 & -2 & 0 & -2 & 0 & 0 & 0 & 2 & 0 & 0 & 0 & -2 & 2 & 2 \\
0 & 0 & 2 & 2 & 0 & -2 & 0 & 0 & 0 & -2 & 0 & 0 & 0 & -2 & 2 & 2 \\
0 & 0 & -2 & 0 & 0 & 2 & -2 & 0 & 2 & 0 & 0 & 0 & 0 & -2 & 2 & 2 \\
0 & 0 & -2 & 0 & 0 & 2 & 2 & 0 & -2 & 0 & 0 & 0 & 0 & -2 & 2 & 2 \\
0 & 0 & -2 & 0 & 0 & -2 & 0 & 0 & 0 & 0 & 2 & -2 & 0 & 2 & 2 & 2 \\
0 & 0 & -2 & 0 & 0 & -2 & 0 & 0 & 0 & 0 & -2 & 2 & 0 & 2 & 2 & 2 \\
0 & 0 & 0 & 0 & 2 & 0 & 0 & -2 & 0 & 0 & 0 & 0 & 0 & 0 & -4 & 4 \\
0 & 0 & 0 & 0 & -2 & 0 & 0 & 2 & 0 & 0 & 0 & 0 & 0 & 0 & -4 & 4 \\
\hline
\end{tabular}

The irreducible characters of $\mathfrak{P}$ will be denoted by $\zeta_{i}, 1 \leqq i \leqq 16$ in the above order.

3. We will require certain facts from the theory of blocks. The terminology is taken from [3]. Let $\mathfrak{5}$ be a group of order $g=p^{a} g^{\prime}$, where $p$ is a fixed prime 
number not dividing $g^{\prime}$. If $B$ is a block of $\left(5\right.$, then the restrictions $\chi_{\mu}^{0}$ of the irreducible characters $\chi_{\mu}$ in $B$ to the $p$-regular classes generate a module $M_{B}$ over $Z$. Any basis $\left\{\phi_{\rho}\right\}$ of $M_{B}$ will be called a basic set $\phi_{B}$ of $B$. We will always assume that when $B$ is the 1 -block of $\left(5\right.$, then the constant function 1 is in $\phi_{B}$. Once a basic set $\phi_{B}$ for $B$ has been chosen, the corresponding decomposition numbers $d_{\mu \rho}$ and Cartan invariants $c_{\rho \sigma}$ of $B$ are defined by

$$
\begin{aligned}
& \chi_{\mu}^{0}=\sum_{\rho} d_{\mu \rho} \phi_{\rho} \text { for } \chi_{\mu} \in B, \phi_{\rho} \in \phi_{B}, \\
& c_{\rho \sigma}=\sum_{\mu} d_{\mu \rho} d_{\mu \sigma} \text { for } \phi_{\rho}, \phi_{\sigma} \in \phi_{B} .
\end{aligned}
$$

$\left(c_{\rho \sigma}\right)$ is the matrix of a positive-definite quadratic form. If $\phi_{B}$ is replaced by another basic set, the form is replaced by an equivalent form. The irreducible modular characters of $\mathfrak{G}$ in $B$ form a basic set, and if $\left(c_{\rho \sigma}\right)$ is the corresponding Cartan matrix, then it is well known that $\sum_{\sigma} c_{\rho \sigma} \phi_{\sigma}(1) \equiv 0\left(\bmod p^{a}\right)$. From this and the above remarks, it follows that the analogous congruence holds for any basic set of $B$.

Let $P$ be a $p$-element of $\left(5\right.$. Let $B^{*}$ run over the blocks of $\mathbb{C}(P)$, and for each $B^{*}$, assume that a basic set $\phi_{B^{*}}$ has been selected. If $V$ runs over $p$-regular elements of $\mathfrak{C}(P)$, the corresponding generalized decomposition numbers $d_{\mu \rho}^{P}$ are defined by

$$
\chi_{\mu}(P V)=\sum d_{\mu \rho}^{P} \phi_{\rho}^{P}(V),
$$

where the sum is over all $\phi_{\rho}^{P} \in \phi_{B^{*}}$ and all $B^{*}$. If $B$ is the 1 -block of $(5$, then the above sum need only be taken over the basic set of the 1-block of $\mathfrak{C}(P)[3$, Theorem 3]. For notational convenience, the column $\left(d_{\mu \rho}^{P}\right)$ will be denoted by $\searrow_{\rho}^{P}$; its $\mu$ th entry $d_{\mu \rho}^{P}$ will be denoted by $\left(\mathfrak{D}_{\rho}^{P}\right)_{\mu}$.

Assume from now on that $B$ and $B^{*}$ are the 1-blocks of $\mathfrak{G}$ and $\mathcal{C}(P)$ respectively. Define for $\chi_{\lambda}, \chi_{\mu} \in B$,

$$
S_{\lambda \mu}^{P}=\frac{1}{c(P)} \sum \chi_{\lambda}(P V) \bar{\chi}_{\mu}(P V)
$$

where $V$ runs over all $p$-regular elements of $\mathscr{C}(P)$. Then by [4] $S_{\lambda \mu}^{P}=\sum_{\rho, \sigma} d_{\lambda \rho}^{P} \tilde{d}_{\mu \sigma}^{P} \gamma_{\rho \sigma}^{P}$, where $\left(\gamma_{\rho \sigma}^{P}\right)$ is the inverse matrix of $C^{P}=\left(c_{\rho \sigma}^{P}\right)$, the matrix of Cartan invariants of $B^{*}$. In particular, if $d=v(P)$, then $p^{d}\left(\gamma_{\rho \sigma}^{P}\right)$ is an integral matrix. Let $Q^{P}$ denote the quadratic form determined by $p^{d}\left(\gamma_{\rho \sigma}^{P}\right) ; Q^{P}$ is positive-definite.

Let $1=P_{1}, P_{2}, \cdots, P_{r}$ be a complete set of representatives for the classes of $p$-elements of $\left(\mathfrak{5}\right.$. Then from the ordinary orthogonality relation $\left(\chi_{\lambda}, \chi_{\mu}\right)_{\mathfrak{G}}=\delta_{\lambda \mu}$ grouped according to the $p$-sections of $\mathfrak{G}$, we have

$$
\sum_{i=1}^{r} S_{\lambda \mu}^{P_{i}}=\delta_{\lambda \mu}= \begin{cases}1 & \lambda=\mu \\ 0 & \lambda \neq \mu\end{cases}
$$


In particular, for $\lambda=\mu, \sum_{i=1}^{r} p^{a} S_{\lambda \lambda}^{P_{i}}=p^{a}$. This latter equation will be referred to as the method of contribution. If $d_{\lambda \rho}^{P}$ is a rational integer for all $\phi_{\rho}^{P}$ in $\phi_{B^{*}}$, and if $v(P)=d$, then $p^{a} S_{\lambda \lambda}^{P}$ is a rational integer $\geqq p^{a-d} m$, where $m$ is the minimum value of the quadratic form $Q^{p}$ for nonzero integral vectors. By [4] we also have the following congruence:

$$
p^{a} S_{\lambda \lambda}^{P} \equiv p^{a} x_{\lambda}^{2} S_{1,1}^{P} \quad\left(\bmod p p^{h} \lambda\right),
$$

where $\chi_{1}$ is the 1-character of $\mathfrak{b}, \chi_{\lambda} \in B, v\left(x_{\lambda}\right)=h_{\lambda}$ is the so-called height of $\chi_{\lambda}$, and $\mathfrak{p}$ is a prime ideal divisor of $p$ in a suitably large algebraic number field. We will refer to this congruence as the $v$ th method of contribution.

If the columns $\grave{D}_{\rho}^{P}$ of the 1-block $B$ have been determined for all $p$-singular sections of $\mathfrak{F}$, and $B$ has $k$ irreducible characters $\chi_{i}$, then any set of integral, linearly independent columns of length $k$ orthogonal to the given $\mathfrak{D}_{\rho}^{P}$ and having a maximal subdeterminant of value 1 is then the set of decomposition numbers of $B$ relative to some basic set $[3, \S 5]$. The corresponding Cartan invariants can then be computed. This technique will be used considerably in the next two sections.

The following remarks may aid in the computations to follow.

(1) If $G \in \mathfrak{z} \mathfrak{G}$ and $G$ has order $p^{\alpha}$, then the $p$-blocks of $\mathfrak{5}$ and $\mathfrak{5} /\langle G\rangle$ are in 1-1 correspondence. The Cartan invariants of a block of $\mathfrak{G}$ are obtained by multiplying the Cartan invariants of the corresponding block of $\mathfrak{5} /\langle G\rangle$ by $p^{\alpha}[3, \S 2]$.

(2) If $\mathfrak{G}$ has a normal $p$-complement, then each $p$-block consists of one modular character. In particular, the Cartan matrix of a block of defect $d$ is $\left(p^{d}\right)[5, \S 2]$.

(3) Let $\mathfrak{D}$ be the dihedral group $\left\langle A, B \mid A^{4}=B^{2}=A B A B=1\right\rangle$ of order 8 . Let $\mathfrak{G}$ be a finite group with $\mathfrak{D}$ as $S_{2}$-subgroup. The classes of involutions in $\mathfrak{D}$ are represented by $A^{2}, B, A B$. Let $C$ be the Cartan matrix of the 1-block for the prime 2. Then one and only one of the following holds: (i) $\mathfrak{5}$ has 3 classes of involutions, $C=(8)$; (ii) $\mathfrak{G}$ has 2 classes of involutions,

$$
A^{2} \sim B \text { or } A^{2} \sim A B, \quad C=\left(\begin{array}{ll}
3 & 1 \\
1 & 3
\end{array}\right) ;
$$

(iii) $\mathfrak{5}$ has 1 class of involutions,

$$
C=\left[\begin{array}{rrr}
2 & 0 & -1 \\
0 & 2 & -1 \\
-1 & -1 & 3
\end{array}\right]
$$

This can be proved by the techniques of this paragraph easily; the details will be omitted.

4. From now on $(\mathfrak{G}$ will be a finite group with no subgroups of index 2 satisfying the assumptions of (2I). By that result the classes of 2-elements fall into one of three cases, which we will denote by I, II, III in the notation of (2I). We 
now compute the Cartan invariants of the 1-block for the 2-singular sections of $\left(5\right.$ and of $\mathfrak{H}=\mathfrak{C}(J)$. Accordingly, if $Y$ is a representative of a 2 -section, $C^{Y}$ and $\widetilde{C}^{Y}$ will denote the matrix of Cartan invariants of the 1-block of $C(Y)$ and $C_{\mathfrak{g}}(Y)$ respectively. In particular, $C^{Y}=\tilde{C}^{Y}$ for $Y=E, F, E^{2}, F^{2}, J$. As we shall see, these matrices are for $\mathfrak{G}$

\begin{tabular}{|c|c|c|c|c|}
\hline$E$ & $F$ & $E^{2}$ & $F^{2}$ & $E N$ \\
\hline 8 & 8 & $\left(\begin{array}{rr}12 & 4 \\
4 & 12\end{array}\right)$ & $\left.\begin{array}{rr}12 & 4 \\
4 & 12\end{array}\right)$ & - \\
\hline 8 & 8 & 32 & 32 & $\left(\begin{array}{ll}6 & 2 \\
2 & 6\end{array}\right)$ \\
\hline 8 & 8 & 32 & 32 & 16 \\
\hline
\end{tabular}

and for $\mathfrak{H}$

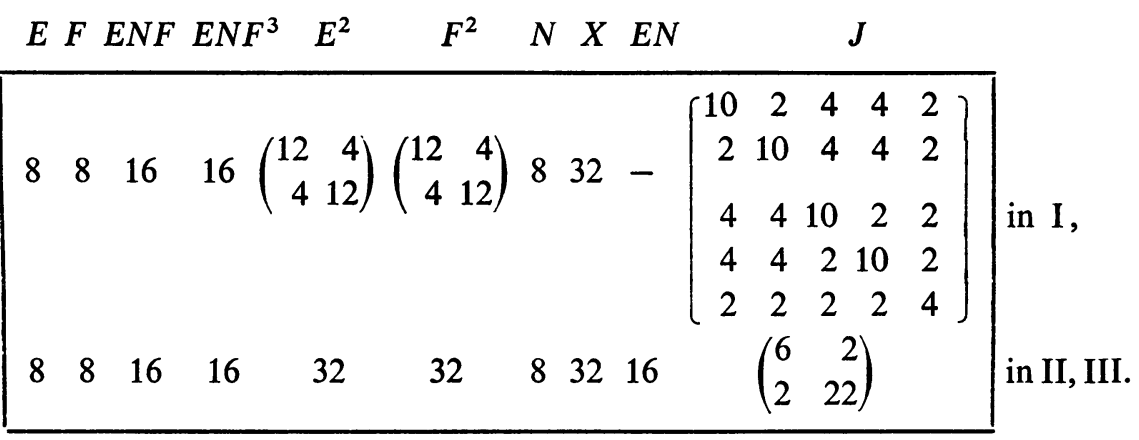

LEMMA (4A). The only fusion in $\mathfrak{H}$ among the 2-elements are the following:

$$
\begin{array}{lll}
E^{2} \sim E N X, & F^{2} \sim N X F, & E F \sim E F^{3} \sim N F \sim E N \sim X \text { in I, } \\
E^{2} \sim E N X, & F^{2} \sim N X F, & E F^{3} \sim N F \sim E N, X \sim E F \quad \text { in II }, \\
E^{2} \sim E N X, & F^{2} \sim N X F, & E F \sim N F \sim E N, \quad X \sim E F^{3} \text { in III. }
\end{array}
$$

In particular, the labeling of the columns in (4.2) is as indicated.

Proof. Since $E N X \sim E^{2}, N X F \sim F^{2}$ in $(5$, and their squares are $J$, these fusions occur in $\mathfrak{S}$. The elements in $\operatorname{ccl}(E N F), \operatorname{ccl}\left(E N F^{3}\right)$ have squares $X$ or $X J$. Hence these classes are not fused in $\mathfrak{H}$ to $E^{2}, F^{2}, E N X, N X F$. We have thus listed the fusions of elements of order 4. If $X \sim E F$ in $\mathfrak{G}$, then $E F^{3} \sim N F \sim E N$ and $X \sim E F$ in $\mathfrak{H}$ by (2F), (2.1). Similarly, if $X \sim E F^{3}$ in $(5)$, then $E F \sim N F \sim E N$ and $X \sim E F^{3}$ in $\mathfrak{H}$. In either case, the focal subgroup $\mathfrak{B}^{*}$ of $\mathfrak{P}$ in $\mathfrak{H}$ contains $\left\langle\mathfrak{P}^{\prime}, E N, N F\right\rangle$ and thus $\left(\mathfrak{P}: \mathfrak{P}^{\#}\right) \leqq 2$. By $(2 \mathrm{G}) \mathfrak{P}^{\#} \neq \mathfrak{P}$, and hence $\operatorname{ccl}(N)$ is isolated in $\mathfrak{S}$. 
COROLlaRY (4B). $\mathfrak{H}$ has a normal subgroup $\tilde{\mathfrak{H}}$ of index 2 , but none of index 4. Moreover, $\tilde{\mathfrak{H}} \cap \mathfrak{P}=\left\langle\mathfrak{P}^{\prime}, E N, N F\right\rangle$. Hence $\mathfrak{H}$ has a linear character $\lambda$ of order 2 with $\lambda(E)=\lambda(F)=\lambda(N)=-1$.

We now turn to the entries of (4.1) and (4.2). $\langle F\rangle,\langle E\rangle$ are $S_{2}$-subgroups of $\mathfrak{C}(F), \mathfrak{C}(E)$ respectively. Since $\mathfrak{C}(F), \mathfrak{C}(E)$ have normal 2-complements, $C^{F}, C^{E}, \tilde{C}^{\boldsymbol{F}}, \tilde{C}^{\boldsymbol{E}}$ are as indicated.

$\mathfrak{C}\left(F^{2}\right)$ has as an $S_{2}$-subgroup the subgroup $\mathbb{Q}=\langle X, F, E N F\rangle$ of order 32 . If residue classes modulo $\left\langle F^{2}\right\rangle$ are denoted by a dot, then $\dot{\mathfrak{Q}}$ has order 8 . If $M=E N F$, then $\dot{M}^{4}=1, \dot{M}^{2}=\dot{X}, \dot{F}^{2}=1, \dot{F} \dot{M} \dot{F}=\dot{M}^{-1}$, and $\dot{\mathfrak{Q}}$ is dihedral. The classes of involutions are represented by $\dot{X}, \dot{F}, \dot{M} \dot{F}$. If $\dot{X} \sim \dot{M} \dot{F}$ in $\widetilde{C}\left(F^{2}\right) /\left\langle F^{2}\right\rangle$, then $X \sim E N$ or $E N X$. This is possible only in I, and indeed, occurs in that case. For since $X \sim E N$ in $I$ in $\mathfrak{5}$, there is a $G \in \mathfrak{F}$ such that $G: E N \rightarrow X$. By $(2 \mathrm{~F})$, (2.1), we may assume $G: F^{2} \rightarrow F^{ \pm 2}$, and since $F^{2} \sim F^{-2}$ in $\mathbb{C}(X)$, we may even assume $G: F^{2} \rightarrow F^{2}$. If $\dot{X} \sim \dot{F}$ in $\mathbb{C}\left(F^{2}\right) /\left\langle F^{2}\right\rangle$, then $X \sim F^{2 i+1}$ for some $i$, which is impossible. The situation for $\mathfrak{C}\left(E^{2}\right)$ is similar; an $S_{2}$-subgroup of $\mathfrak{C}\left(E^{2}\right)$ is $\langle X, E, E N F\rangle$. Thus $C^{E^{2}}, C^{F^{2}}, \tilde{C}^{E^{2}} \tilde{C}^{F^{2}}$ are as indicated.

In cases II, III, $\mathfrak{C}(E N)$ has $\langle E N\rangle \times\left\langle F^{2}, N F\right\rangle$ as $S_{2}$-subgroup, where $\mathfrak{Q}=\left\langle F^{2}, N F\right\rangle$ is dihedral of order 8 . If residue classes modulo $\langle E N\rangle$ are denoted by a dot, then the classes of involutions of $\dot{\mathfrak{Q}}$ are represented by $\dot{J}, N \dot{F}$, $N \dot{F}^{3}$. Suppose we are in case II. If $\dot{J} \sim N \dot{F}^{3}$, then $J \sim N F^{3}$ or $J \sim(E N)\left(N F^{3}\right)=E F^{3}$ in $\mathfrak{C}(E N)$, which is impossible. If $\dot{J} \sim N \dot{F}$, then $J \sim N F$ or $J \sim E F$ in $\mathscr{C}(E N)$, and only the latter can occur in case II. To see that this does occur, replace $E N$ by the element $Y$ in $\operatorname{ccl}\left(E F^{3}\right)$ which is fixed by the element $B$ in $(2 E)$. In $\mathscr{C}(Y) /\langle Y\rangle$, $B$ fuses two classes of involutions, and so

$$
C^{E N}=\left(\begin{array}{ll}
6 & 2 \\
2 & 6
\end{array}\right)
$$

Suppose we are in III. If $\dot{J} \sim \dot{N} F$, then $J \sim N F$ or $J \sim E F$, which is impossible. If $\dot{J} \sim N \dot{F}^{3}$, then necessarily $J \sim E F^{3}$ in $\widetilde{C}(E N)$. To see that this does not occur,

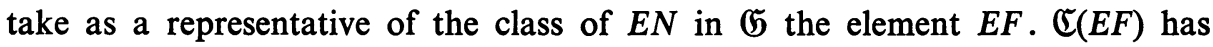
$\langle E F\rangle \times\langle N X F, X\rangle$ as $S_{2}$-subgroup. Moreover, if $J \sim E F^{3}$ in $\mathscr{C}(E N)$, then $J \sim X$ in $\mathfrak{C}(E F)$, and there is an $A \in \mathfrak{G}$ such that $A: X \rightarrow J, E F \rightarrow E F$. On the other hand, by (2E) there is a $B \in \mathfrak{G}$ such that $B: J \rightarrow X, N_{1} \rightarrow \operatorname{ccl}(E F)$, where $N_{1} \in \operatorname{ccl}(N)$. Since $E F$ has 4 conjugates in $\mathbb{C}_{\beta}(X)$, we may assume $B: N_{1} \rightarrow E F$. Then $B A: J \rightarrow J, N_{1} \rightarrow E F$. If $\mathfrak{P}^{*}$ is the focal subgroup of $\mathfrak{P}$ in $\mathscr{C}(J)$, then $E N F \in \mathfrak{P}^{\#}$, which contradicts (4B). Thus $J \approx E F^{3}$ in $\mathscr{C}(E N)$, and $C^{E N}=(16)$. This proves the validity of (4.1).

In $\mathfrak{T} /\langle J\rangle$ let small letters denote residue classes of corresponding capital letters. By (4A) the following are representatives of the classes of 2-elements of $\mathfrak{T} /\langle J\rangle$.

$$
e, f, \text { enf }, e^{2}, f^{2}, x, e n, n, 1
$$


except that in case $\mathrm{I}$, en is to be omitted. For $Y \in \mathfrak{H}$, let $\mathfrak{C}^{0}(Y)$ be the inverse image in $\mathfrak{H}$ of the centralizer $\dot{\mathbb{C}}(y)$ of $y$ in $\mathfrak{H} /\langle J\rangle$. Then $\mathfrak{C}_{\mathfrak{S}}(Y) \checkmark \mathfrak{C}^{0}(Y)$, and the index is 1 or 2 . In particular, if $\mathfrak{C}(Y)$ has a normal 2-complement, so do $\mathfrak{C}_{\mathfrak{f g}}(Y)$ and $\mathfrak{C}^{0}(Y)$. If $y$ is a representative of a 2 -section of $\mathfrak{H} /\langle J\rangle$, let $\dot{C}^{y}$ be the matrix of Cartan invariants of the 1-block of $\dot{\mathscr{C}}(y)$. We then have $\dot{C}^{e}=\dot{C}^{f}=(8)$. $\dot{\mathscr{C}}($ enf $)$ has $\langle$ enf $\rangle \times\left\langle f^{2}\right\rangle$ as an $S_{2}$-subgroup, so that $\dot{\mathscr{C}}($ enf $)$ has a normal 2-complement, and $\dot{C}^{e n f}=(8)$. For $\dot{\mathfrak{C}}\left(e^{2}\right)$ and $\dot{\mathfrak{C}}\left(f^{2}\right)$ the above remarks apply in cases II, III, so that $\dot{C}^{e^{2}}=\dot{C}^{f^{2}}=(32)$ in these cases. In case I $\dot{\mathscr{C}}\left(f^{2}\right) \mid\left\langle f^{2}\right\rangle$ has as an $S_{2}$-subgroup $\mathfrak{Q} \times\langle N\rangle\left(\bmod \left\langle F^{2}\right\rangle\right)$, where $\mathfrak{Q}=\langle X, F, E N F\rangle$. $\dot{\mathfrak{C}}\left(f^{2}\right) /\left\langle f^{2}\right\rangle$ has a normal subgroup of index 2 with dihedral $S_{2}$-subgroups. Moreover, the center of $\mathfrak{Q} \times\langle N\rangle\left(\bmod \left\langle F^{2}\right\rangle\right)$ is not contained in this subgroup From our earlier remarks and [5] (3B), it follows that

$$
C^{f^{2}}=\left(\begin{array}{rr}
12 & 4 \\
4 & 12
\end{array}\right)
$$

Similarly,

$$
\dot{C}^{e^{2}}=\left(\begin{array}{rr}
12 & 4 \\
4 & 12
\end{array}\right)
$$

$\dot{\mathfrak{C}}(x)$ has a normal 2-complement by the remark following $(2 \mathrm{G})$, so that $\dot{C}^{x}=(32)$. $\dot{\mathfrak{C}}(n)$ has $\left\langle n, e^{2}, f^{2}\right\rangle$ as $S_{2}$-subgroup, elementary abelian of order 8 . Thus $\dot{\widetilde{C}}(n) /\langle n\rangle$ has an $S_{2}$-subgroup of type $(2,2)$, whose involutions modulo $\langle n\rangle$ are $e^{2}, f^{2}, x=e^{2} f^{2}$. If these are fused in $\dot{\mathfrak{C}}(n) /\langle n\rangle$, then $e^{2}, f^{2}, x$ are fused in $\dot{\mathcal{C}}(n)$ which is impossible. Thus $\dot{\mathfrak{C}}(n)$ has a normal 2-complement, and $\dot{C}^{n}=(8)$. Finally, in cases II, III, $\mathfrak{C}_{\mathfrak{H}}(E N)$ has $\langle E N\rangle \times\left\langle F^{2}, N F\right\rangle$ as an $S_{2}$-subgroup, and there are no fusions of involutions in $\mathfrak{C}_{\mathfrak{g}}(E N) /\langle E N\rangle$, since $J$ is isolated in $\mathfrak{H}$. Thus $\mathfrak{C}_{\mathfrak{g}}(E N)$ and $\dot{\mathfrak{C}}(e n)$ have normal 2-complements, and $\dot{C}^{e n}=(16)$. Summarizing ithe calculations of this paragraph, we have

\begin{tabular}{|c|c|c|c|c|c|c|c|c|}
\hline$e$ & $f$ & enf & $e^{2}$ & $f^{2}$ & $x$ & $n$ & en & \\
\hline 8 & 8 & 8 & $\left(\begin{array}{rr}12 & 4 \\
4 & 12\end{array}\right)$ & $\left(\begin{array}{rr}12 & 4 \\
4 & 12\end{array}\right)$ & 32 & 8 & - & in $\mathrm{I}$, \\
\hline 8 & 8 & 8 & 32 & 32 & 32 & 8 & 16 & in II, III. \\
\hline
\end{tabular}

The entries of (4.2) are now clear, except for those under $J$.

Let $\chi_{1}$ be the 1-character of $\mathfrak{H}$. Then $d_{1,1}^{e}=d_{1,1}^{f}=d_{1,1}^{e n f}=1$. The existence of the character $\lambda$ of (4B) shows that the nonzero entries under $e, f$, enf, $n$ are four 1's and four -1 's. These nonzero generalized decomposition numbers exhaust the characters of height 0 , and hence the 1-block $\widetilde{B}$ of $\mathfrak{H}$ has exactly 8 characters of height 0 . After a rearrangement if necessary, we may assume by the orthogonality relations that $\chi_{i}$ for $1 \leqq i \leqq 8$ are as below: 


$$
\begin{array}{|rrrr}
e & f & \text { enf } & n \\
\hline 1 & 1 & 1 & 1 \\
-1 & -1 & -1 & -1 \\
1 & 1 & -1 & -1 \\
-1 & -1 & 1 & 1 \\
1 & -1 & 1 & -1 \\
-1 & 1 & -1 & 1 \\
1 & -1 & -1 & 1 \\
-1 & 1 & 1 & -1 \\
\hline
\end{array}
$$

Cases II, III. For $1 \leqq \mu \leqq 8, d_{\mu 1}^{e n}$ are odd integers occurring in pairs; hence they are \pm 1 , since $\Sigma_{\mu}\left(d_{\mu 1}^{e n}\right)^{2}=16$. The remaining nonzero entries in $D_{1}^{e n}$ are even integers whose squares add up to 8 . Hence these are \pm 2 , and we may assume they occur for $\chi_{9}, \chi_{10}$. If there is a $\chi_{11}$ in the 1-block $\dot{B}$ of $\mathfrak{g} /\langle\mathrm{J}\rangle$, consider $\chi_{11}$ restricted to $\left\langle e n, n f^{3}\right\rangle$ in II, to $\langle e n, n f\rangle$ in III. These subgroups are abelian of type $(2,2)$, and their involutions are fused in $\mathfrak{H}$. Hence $3 \chi_{11}(e n) \equiv \chi_{11}(1)(\bmod 4)$. Since $\chi_{11}(e n)=0, \chi_{11}$ has height $\geqq 2$, and thus each of the numbers $\chi_{11}\left(e^{2}\right)$, $\chi_{11}\left(f^{2}\right), \chi_{11}(x)$ is nonzero and divisible by 4 by the $v$ th method of contribution. But then $4^{2}+4^{2}+4^{2}>32$, and this is impossible by the method of contribution applied to $\chi_{11}, \dot{B}$ then has exactly 10 irreducible characters, and therefore 2 modular characters for the 1-section. For $i=9,10, \chi_{i}\left(e^{2}\right), \chi_{i}\left(f^{2}\right), \chi_{i}(x)= \pm 2$, since \pm 6 is too large. The matrix of generalized decomposition numbers for $\dot{B}$ can now be essentially completed. A $\chi \in \dot{B}$ cannot assume \pm 3 more than once on $e^{2}, f^{2}, x$ by the method of contribution. Orthogonality relations are then sufficient to compute the remaining entries with the uncertainties indicated below. We omit the details.

\begin{tabular}{|c|c|c|c|c|c|c|c|c|c|}
\hline$e$ & $f$ & enf & $e^{2}$ & $f^{2}$ & $x$ & $n$ & en & & \\
\hline 1 & 1 & 1 & 1 & 1 & 1 & 1 & 1 & 1 & 0 \\
\hline-1 & -1 & -1 & 1 & 1 & 1 & -1 & 1 & 1 & 0 \\
\hline 1 & 1 & -1 & & & & -1 & $\tau_{1}$ & 0 & $\tau_{1}$ \\
\hline-1 & -1 & 1 & & & & 1 & $\tau_{1}$ & 0 & $\tau_{1}$ \\
\hline 1 & -1 & 1 & & & & -1 & $\tau_{2}$ & 0 & $\tau_{2}$ \\
\hline-1 & 1 & -1 & $\mathfrak{S}_{\alpha}$ & $\mathfrak{S}_{\beta}$ & $\mathfrak{S}_{\gamma}$ & 1 & $\tau_{2}$ & 0 & $\tau_{2}$ \\
\hline 1 & -1 & -1 & & & & 1 & $\tau_{3}$ & 0 & $\tau_{3}$ \\
\hline-1 & 1 & 1 & & & & -1 & $\tau_{3}$ & 0 & $\tau_{3}$ \\
\hline 0 & 0 & 0 & $2 \omega_{1}$ & $2 \omega_{1}$ & $2 \omega_{1}$ & 0 & $-2 \omega_{1}$ & 0 & $2 \omega_{1}$ \\
\hline 0 & 0 & 0 & $2 \omega_{2}$ & $2 \omega_{2}$ & $2 \omega_{2}$ & 0 & $2 \omega_{2}$ & $-\omega_{2}$ & $-\omega_{2}$ \\
\hline
\end{tabular}

Here $\tau_{i}, \omega_{i}= \pm 1,\{\alpha, \beta, \gamma\}$ is a permutation of $\{1,2,3\}$, and 


$$
\mathfrak{S}_{1}=\begin{array}{r}
-3 \tau_{1} \\
-3 \tau_{1} \\
\tau_{2} \\
\tau_{2} \\
\tau_{3} \\
\tau_{3}
\end{array}, \quad \mathfrak{S}_{2}=\begin{array}{r}
\tau_{1} \\
\tau_{1} \\
-3 \tau_{2} \\
-3 \tau_{2} \\
\tau_{3} \\
\tau_{3}
\end{array}, \quad \mathfrak{S}_{3}=\begin{array}{r}
\tau_{1} \\
\tau_{1} \\
\tau_{2} \\
\tau_{2} \\
-3 \tau_{3} \\
-3 \tau_{3}
\end{array}
$$

The columns under 1 are determined by the methods of $\S 3 . \tilde{C}^{J}$ is then

$$
\left(\begin{array}{rr}
6 & 2 \\
2 & 22
\end{array}\right)
$$

If $f_{1}^{J}, f_{2}^{J}$ are the degrees of the functions in the corresponding basic set, then $f_{1}^{J}=1$ since $\phi_{1}^{J}$ is the constant 1 . Since $6 f_{1}^{J}+2 f_{2}^{J} \equiv 0(\bmod 64)$, we see that $f_{2}^{J} \equiv-3(\bmod 32)$. This will be important later on.

The matrix of generalized decomposition numbers for the 1-block $\widetilde{B}$ of $\mathfrak{H}$ in cases II, III can now be completed. A calculation of the $S_{2}$-subgroups shows that except for $E N F$ and $E N F^{3}$, all entries under the various representatives $\neq J$ of the 2-singular sections from characters faithful on $J$ are 0 . Moreover, the nonzero entries for $E N F, E N F^{3}$ are rational even integers whose squares add up to 8 . This implies the existence of $\chi_{11}, \chi_{12}$ as indicated below. Since there are at least 13 columns, there is one more character $\chi_{13}$. The entries of $\chi_{11}, \chi_{12}, \chi_{13}$ under $J$ and 1 are easily determined by orthogonality relations. The arrangement of the signs is achieved by interchanging $\chi_{11}$ and $\chi_{12}$ and replacing either by its negative if necessary. The matrix of generalized decomposition numbers for $\widetilde{B}$ in cases II, III is

\begin{tabular}{|c|c|c|c|c|c|c|c|c|c|c|c|}
\hline$E$ & $F$ & $E^{2}$ & $F^{2}$ & $E N F$ & $E N F^{3}$ & $X$ & $N$ & $E N$ & J & $J$ & 1 \\
\hline 1 & 1 & 1 & 1 & 1 & 1 & 1 & 1 & 1 & 1 & 0 & 0 \\
\hline-1 & -1 & 1 & 1 & -1 & -1 & 1 & -1 & 1 & 1 & 0 & 1 \\
\hline 1 & 1 & & & -1 & -1 & & -1 & $\tau_{1}$ & 0 & $\tau_{1}$ & $\begin{array}{ll}0 & \tau_{1}\end{array}$ \\
\hline-1 & -1 & & & 1 & 1 & & 1 & $\tau_{1}$ & 0 & $\tau_{1}$ & $\tau_{1}$ \\
\hline 1 & -1 & $\mathfrak{S}_{\alpha}$ & $\mathfrak{S}_{\beta}$ & 1 & 1 & $\mathfrak{S}_{\gamma}$ & -1 & $\tau_{2}$ & 0 & $\tau_{2}$ & $\tau_{2}$ \\
\hline-1 & 1 & & & -1 & -1 & & 1 & $\tau_{2}$ & 0 & $\tau_{2}$ & $\tau_{2}$ \\
\hline 1 & -1 & & & -1 & -1 & & 1 & $\tau_{3}$ & 0 & $\tau_{3}$ & $\tau_{3}$ \\
\hline-1 & 1 & & & 1 & 1 & & -1 & $\tau_{3}$ & 0 & $\tau_{3}$ & $\tau_{3}$ \\
\hline 0 & 0 & $2 \omega_{1}$ & $2 \omega_{1}$ & 0 & 0 & $2 \omega_{1}$ & 0 & $-2 \omega_{1}$ & 0 & $2 \omega_{1}$ & $02 \omega_{1}$ \\
\hline 0 & 0 & $2 \omega_{2}$ & $2 \omega_{2}$ & 0 & 0 & $2 \omega_{2}$ & 0 & $2 \omega_{2}$ & $-\omega_{2}$ & $-\omega_{2}$ & $-\omega_{2}-\omega_{2}$ \\
\hline 0 & 0 & 0 & 0 & 2 & -2 & 0 & 0 & 0 & -1 & 1 & $1-1$ \\
\hline 0 & 0 & 0 & 0 & -2 & 2 & 0 & 0 & 0 & -1 & 1 & $1-1$ \\
\hline 0 & 0 & 0 & 0 & 0 & 0 & 0 & 0 & 0 & -1 & -3 & 1 \\
\hline
\end{tabular}


where $\omega_{i}, \tau_{i}= \pm 1$, and $\mathfrak{S}_{\alpha}, \mathfrak{S}_{\beta}, \mathfrak{S}_{\gamma}$ are as in (4.6). $C^{J}$ is then

$$
\left(\begin{array}{rr}
6 & 2 \\
2 & 22
\end{array}\right)
$$

as indicated in (4.2).

Case I. Let $\dot{B}$ be the 1-block of $\mathfrak{S} /\langle J\rangle$. Then no $\chi_{i} \in \dot{B}$ is zero on $x$, since $x$ is in the center of $\mathfrak{P} \mid\langle J\rangle$. If $\chi_{i}(x)= \pm 3$ for some $1 \leqq i \leqq 8$, then this occurs at least twice, so that $\sum_{i=1}^{8} \chi_{i}(x)^{2} \geqq 24$. Since the remaining $\chi_{i} \in \dot{B}$ have height $\geqq 1$, this would imply that $\dot{B}$ has $\leqq 10$ characters, which is impossible. Thus we may assume $\chi_{i}(x)=\chi_{i+1}(x)=\tau_{i}$ for $i=3,5,7, \tau_{i}= \pm 1$. The $\chi_{i} \in \dot{B}$ for $i \geqq 9$ cannot have height $\geqq 2$; otherwise the contribution from $e^{2}, f^{2}, x$ would be too large. Hence these $\chi_{i}$ have height 1 , and necessarily $\chi_{i}(x)=2 \tau_{i}, \tau_{i}= \pm 1$. In particular, $\dot{B}$ has 14 characters and 5 functions in the basic set from the 1 -section. The quadratic forms $Q^{E^{2}}$ and $Q^{F^{2}}$ associated to $\mathfrak{C}\left(E^{2}\right)$ and $\mathfrak{C}\left(F^{2}\right)$ are $3 u^{2}-2 u v+3 v^{2}=2 u^{2}$ $+2 v^{2}+(u-v)^{2}$, which has a minimum of 3 for nonzero rational integral vectors. If a $\chi_{i} \in \dot{B}$ has height 0 , then the sum of the contributions from $E^{2}, F^{2}, J, 1$ must be $64-34=30$. We express this as

$$
2 Q^{E^{2}}+2 Q^{F^{2}}+Q^{J}+Q^{1}=30
$$

Now $Q^{J}, Q^{1} \geqq 1$. Since $d_{i 1}^{E^{2}}-d_{i 2}^{E^{2}}$ and $d_{i 1}^{F^{2}}-d_{i 2}^{F^{2}}$ are necessarily odd, $Q^{E^{2}}$ and $Q^{F^{2}}$ are $\leqq 11$. If $(u, v)$ are the decomposition numbers of $\chi_{i}$ under $E^{2}$ or $F^{2}$, and if we allow for a possible change of $\operatorname{sign}(u, v) \rightarrow(-u,-v)$ and a possible interchange $(u, v) \rightarrow(v, u)$, then the only possible values for $(u, v)$ are $(1,0)$ and $(2,1)$. If $\chi_{i} \in \dot{B}$ has height 1 , then $u+v \equiv 2(\bmod 4)$. The method of contributions implies that $Q^{E^{2}}$ and $Q^{F^{2}} \leqq 30$. With the same conventions as before, the only possible values for $(u, v)$ are $(1,1)$ and $(2,0)$. Consider the entries under $E^{2}$ for all $\chi_{i} \in \dot{B}$. If there are $a, b, c, d$ rows of type $(1,0),(2,1),(1,1),(2,0)$ respectively, then from the equality $\Sigma u^{2}+\Sigma v^{2}=24$, we have $a+b=8, c+d=6, a+5 b+2 c+4 d=24$. Thus $b=0, d=2$. The entries can then be determined by the orthogonality relations. Similarly so for the entries under $F^{2}$. The generalized decomposition numbers for $\dot{B}$ for the 2-singular sections of $\mathfrak{H} /\langle J\rangle$ have now been determined, and are as below. There are then 6 characters of $\widetilde{B}$ faithful on $\langle J\rangle$. Two of them, say $\chi_{15}, \chi_{16}$, are nonzero on $E N F$, $E N F^{3}$. The columns under $J$ and 1 can then be found by the methods of $\S 3$. The matrix for $\widetilde{B}$ in case $I$ is (4.8), where $\tau_{i}= \pm 1$. $\widetilde{C}^{J}$ is then as in (4.2).

5. In addition to the assumptions made on $\mathfrak{5}$ on $\$ 4$, we also assume $\mathfrak{6}$ has more than one class of involutions. This excludes case I.

LEMMA (5A). Let $\chi$ be a character of $(5$. Then

(i) $\chi(E) \equiv \chi(F)(\bmod 2)$,

(ii) $\chi\left(E^{2}\right) \equiv \chi\left(F^{2}\right) \equiv \chi(E N) \equiv \chi(J)(\bmod 4)$,

(iii) $\chi(J) \equiv 2 \chi(E)-\chi\left(F^{2}\right) \equiv 2 \chi(F)-\chi\left(E^{2}\right)(\bmod 8)$,

(iv) $-2 \chi(E)-2 \chi(F)+\chi\left(F^{2}\right)+\chi\left(E^{2}\right)+2 \chi(E N) \equiv 0(\bmod 8)$. 


\begin{tabular}{|c|c|c|}
\hline 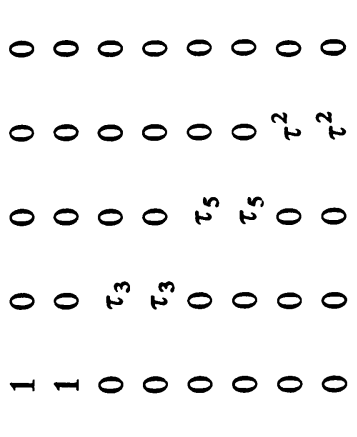 & 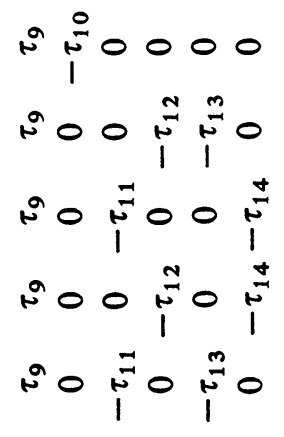 & 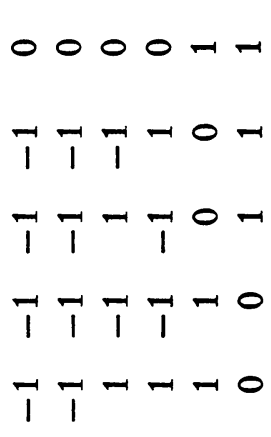 \\
\hline 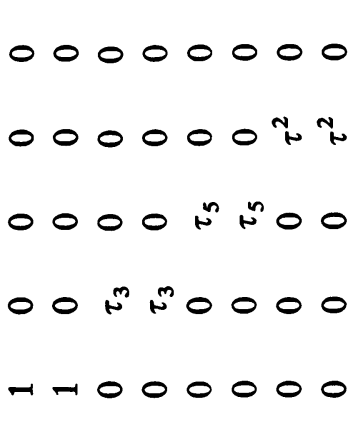 & 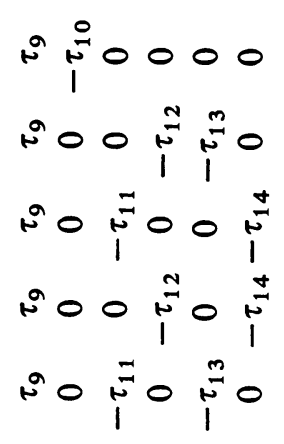 & 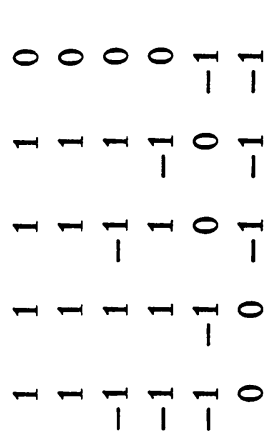 \\
\hline 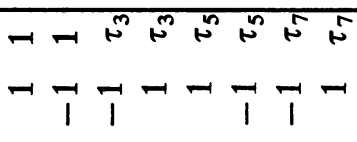 & 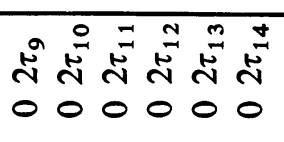 & 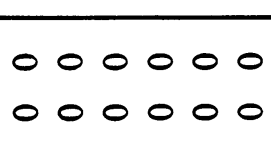 \\
\hline 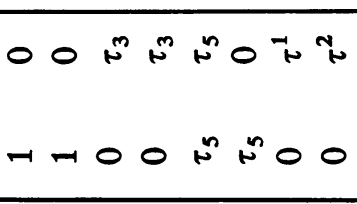 & 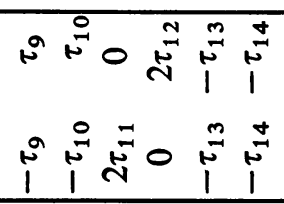 & $\begin{array}{l}000000 \\
000000\end{array}$ \\
\hline 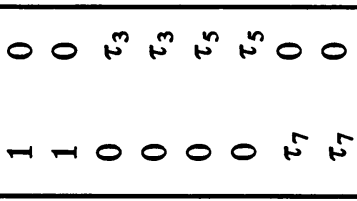 & 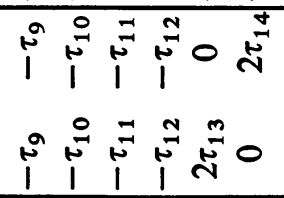 & $\begin{array}{l}000000 \\
000000\end{array}$ \\
\hline 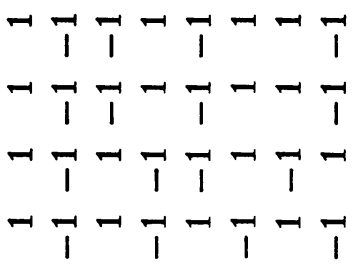 & $\begin{array}{l}000000 \\
000000 \\
000000 \\
000000\end{array}$ & $\begin{array}{lllllll}0 & 0 & 0 & 0 & 0 & 0 \\
0 & 0 & 0 & 0 & 0 & 0 \\
N & N & 0 & 0 & 0 & 0 \\
N & 0 & 0 & 0 & 0 \\
N & n\end{array}$ \\
\hline
\end{tabular}


Proof. (i) is obvious, since $\chi(E)$ and $\chi(F)$ are rational integers congruent to $\chi(1)(\bmod 2)$. The others follow by restricting $\chi$ to $\mathfrak{P}$ and computing $(\chi, \phi) \mathfrak{P}$, where $\phi$ is a suitable generalized character of $\mathfrak{P}$. For (ii) take $\phi$ to be respectively $\zeta_{15}-\zeta_{16}, \zeta_{9}-\zeta_{10}, \zeta_{13}-\zeta_{14}$ in the notation of (2.2). For (iii) take $\phi$ to be $\zeta=\zeta_{1}-\zeta_{5}$, which yields $2 \chi(E)+\chi\left(F^{2}\right)+2 \chi\left(E^{2}\right)+2 \chi(E N)+\chi(J) \equiv 0(\bmod 8)$. By (ii) $2 \chi\left(F^{2}\right) \equiv 2 \chi\left(E^{2}\right), 2 \chi(E N) \equiv 2 \chi(J)(\bmod 8)$. Substitution of these gives the first part of (iii). The second part is proved similarly. For (iv) take $\phi=2 \zeta_{1}-\zeta_{9}$, and use the congruence $2 \chi(E N) \equiv 2 \chi(J)(\bmod 8)$.

Let $B$ be the 1-block of $(5$. If $Y$ is a representative of a 2 -singular section of $\mathbb{5}$, we assume a basic set $\phi_{B}=\left\{\phi_{\rho}^{Y}\right\}$ has been chosen for the 1-block of $\mathcal{C}(Y)$ such that the corresponding Cartan invariants are as in (4.1), and for $Y=J$, as in (4.2). Denote by $f_{\rho}^{Y}$ the degree of $\phi_{\rho}^{Y} ; \phi_{1}^{Y}$ is always the 1-character of $\mathbb{C}(Y$,$) ,$ so that $f_{1}^{Y}=1$. In particular, $f_{2}^{E N} \equiv-3(\bmod 8)$ in case II, and $f_{2}^{J} \equiv-3(\bmod$ 32). The corresponding columns of generalized decomposition numbers are then denoted by $\mathfrak{D}_{\rho}^{Y}$, except that in the case where $\mathfrak{C}(Y)$ has a normal 2-complement and there is but one such column, we will drop the index 1 . For $\chi_{i} \in B$, $\chi_{i}(Y)=\Sigma_{\rho}\left(\mathfrak{D}_{\rho}^{Y}\right)_{i} f_{\rho}^{Y}$, where $\left(\mathfrak{D}_{\rho}^{Y}\right)_{i}$ is the entry $d_{i \rho}^{Y}$.

Define the following columns of rational integers, indexed by the $\chi_{i} \in B$.

$$
\begin{aligned}
& \mathfrak{a}=\frac{\mathfrak{D}^{F}-\mathfrak{d}^{E}}{2}, \\
& \mathfrak{b}=\frac{\mathfrak{D}^{F}+\mathfrak{D}^{E}}{2}, \\
& \mathfrak{w}= \begin{cases}\frac{\mathfrak{d}_{1}^{E N}+\mathfrak{d}_{2}^{E N}-\mathfrak{D}^{F^{2}}}{4} & \text { in II, } \\
\frac{\mathfrak{D}^{E N}-\mathfrak{D}^{F^{2}}}{4} & \text { in III, }\end{cases} \\
& \mathfrak{S}=\frac{\mathfrak{d}_{1}^{J}-3 \mathfrak{d}_{2}^{J}+\mathfrak{d}^{F^{2}}-2 \mathfrak{d}^{E}}{8}
\end{aligned}
$$

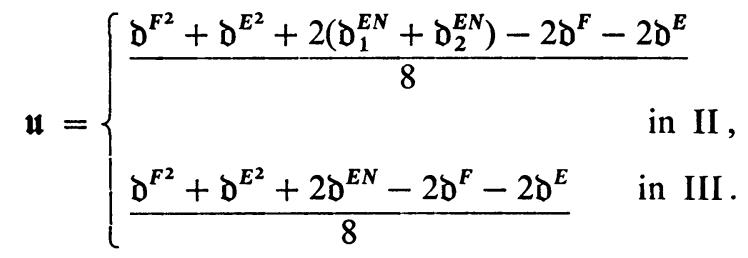

$$
\begin{aligned}
& \mathfrak{U}=\frac{\mathfrak{D}^{F^{2}}-\grave{D}^{E^{2}}}{4},
\end{aligned}
$$

That these columns are rational integral follows from (5A) and the congruences $f_{2}^{E N} \equiv-3(\bmod 8), f_{2}^{J} \equiv-3(\bmod 32)$. The inner products of these columns and the ones indicated are

\begin{tabular}{c|rrrrrrr|r|r|r|} 
& $\mathfrak{a}$ & $\mathfrak{b}$ & $\mathfrak{w}$ & $\mathfrak{S}$ & $\mathfrak{U}$ & $\mathfrak{u}$ & $\mathfrak{d}_{1}^{J}$ & \multicolumn{1}{c}{$\mathfrak{d}^{E N}$} & $\mathfrak{d}_{1}^{E N}$ & $\mathfrak{d}_{2}^{E N}$ \\
\hline $\mathfrak{a}$ & 4 & 0 & 0 & 1 & 0 & 0 & 0 & 0 & 0 & 0 \\
$\mathfrak{b}$ & 0 & 4 & 0 & -1 & 0 & -2 & 0 & 0 & 0 & 0 \\
$\mathfrak{w}$ & 0 & 0 & 3 & -1 & -2 & 0 & 0 & 4 & 2 & 2 \\
$\mathfrak{S}$ & 1 & -1 & -1 & 4 & 2 & 1 & 0 & 0 & 0 & 0 \\
$\mathfrak{U}$ & 0 & 0 & -2 & 1 & 4 & 0 & 0 & 0 & 0 & 0 \\
$\mathfrak{u}$ & 0 & -2 & 0 & 1 & 0 & 3 & 0 & 4 & 2 & 2 \\
\hline
\end{tabular}

where $\mathfrak{D}^{E N}$ occurs in III; $\mathfrak{D}_{1}^{E N}, \mathfrak{D}_{2}^{E N}$ in II. By $(5 \mathrm{~A})$,(iii) and the congruence 
$\chi(E) \equiv \chi\left(E^{2}\right)(\bmod 2)$, it follows that $\mathfrak{A} \equiv \mathfrak{a}(\bmod 2)$. We note that $\mathfrak{a}$ and $\mathfrak{b}$ are orthogonal to the column $\chi(1)$ of degrees.

We now determine the entries of these columns. $\mathfrak{w}$ and $\mathfrak{u}$ have norm 3 , and thus have 3 entries $\pm 1 . \mathfrak{a}, \mathfrak{b}, \mathfrak{S}, \mathfrak{A}$ have norm 4 , and since $\mathfrak{a} \mathfrak{S}=1, \mathfrak{b} \mathfrak{S}=-1$, each of these must have 4 entries \pm 1 . We can choose five characters $\chi$ such that by replacing $\chi$ by $-\chi$ if necessary, we have

\begin{tabular}{|rrrr|}
$\mathfrak{w}$ & $\mathfrak{U}$ & $\mathfrak{a}$ & $\mathfrak{b}$ \\
\hline 1 & 0 & 0 & $b_{1}$ \\
1 & -1 & 1 & $b_{2}$ \\
1 & -1 & -1 & $b_{3}$ \\
0 & 1 & 1 & $b_{4}$ \\
0 & 1 & -1 & $b_{5}$ \\
\hline
\end{tabular}

By orthogonality $b_{3}=b_{4}, b_{2}=b_{5}, b_{1}=-b_{2}-b_{3}$. Suppose $b_{i} \neq 0$ for some $2 \leqq i \leqq 5$. Then one of $\chi_{i}(E), \chi_{i}(F)$ is \pm 2 , and the other is 0 ; say that $\chi_{i}(E)= \pm 2$, $\chi_{i}(F)=0$. If $\chi_{i}$ has height 1 , then by $(5 \mathrm{~A}),(\mathrm{ii}) \chi_{i}\left(F^{2}\right) \equiv \chi_{i}\left(E^{2}\right) \equiv \chi_{i}(E N) \equiv 2(\bmod 4)$. The contribution to $\chi_{i}$ from $E, F^{2}, E^{2}, E N$ is then 64 , which is too large. If $\chi_{i}$ has height $\geqq 2$, then $\chi_{i}\left(F^{2}\right) \equiv \chi_{i}\left(E^{2}\right) \equiv 0 \quad(\bmod 4)$. But (5A), (iii) implies $\chi_{i}(J)+\chi_{i}\left(F^{2}\right) \equiv 4(\bmod 8)$ and the same congruence with the roles of $E$ and $F$ interchanged implies that $\chi_{i}(J)+\chi_{i}\left(E^{2}\right) \equiv 0(\bmod 8)$. Thus one of $\chi_{i}\left(E^{2}\right), \chi_{i}\left(F^{2}\right)$ is nonzero, and the method of contribution again leads to a contradiction. The argument for the case $\chi_{i}(E)=0, \chi_{i}(F)= \pm 2$ is similar. Thus $b_{i}=0$ for $2 \leqq i \leqq 5$, and $b_{1}=0$ as well.

We now rearrange the characters in $B$ so that $\chi_{1}$ is the 1-character and the others are as indicatedybelow:

\begin{tabular}{|rrrrr|}
$\mathfrak{b}$ & $\mathfrak{a}$ & $\mathfrak{U}$ & $\mathfrak{w}$ & $\mathfrak{u}$ \\
\hline 1 & 0 & 0 & 0 & 0 \\
1 & 0 & 0 & 0 & -1 \\
1 & 0 & 0 & 0 & -1 \\
1 & 0 & 0 & 0 & 0 \\
0 & 1 & -1 & 1 & 0 \\
0 & -1 & -1 & 1 & 0 \\
0 & 1 & 1 & 0 & 0 \\
0 & -1 & 1 & 0 & 0 \\
0 & 0 & 0 & 1 & 0 \\
0 & 0 & 0 & 0 & 1 \\
\hline
\end{tabular}

The entries of $\mathfrak{u}$ are determined easily from (5.1). Since $\mathfrak{A}$ is known, the method of contributions implies that $\left(D^{E^{2}}, \mathfrak{D}^{F^{2}}\right)_{i}= \pm(1,1)$ for $1 \leqq i \leqq 4$, and $\left(D^{E^{2}}, \delta^{F^{2}}\right)_{i}= \pm(1,-3)$ or $\pm(-3,1)$ for $5 \leqq i \leqq 8$. Furthermore, knowledge of 
$\mathfrak{u}$ and $\mathfrak{w}$ imply that $\left(\mathfrak{D}^{E^{2}}, \mathfrak{D}^{F^{2}}\right)_{i}=(-2,-2)$ for $i=9$, and $(2,2)$ for $i=10$. The signs of $\left(D^{E^{2}}, \downarrow^{F^{2}}\right)_{i}$ for $i=2,3,4$ can be determined by the equation $u \delta^{E^{2}}=4$. Since $D^{E^{2}}, D^{F^{2}}$ have norm 32 , each has exactly two \pm 3 's for $5 \leqq i \leqq 8$. The products $\mathfrak{w} \mathfrak{D}^{E^{2}}=0, \mathfrak{w} \mathfrak{D}^{F^{2}}=-8$ determine the signs completely. If the characters are arranged as in (5.2), then

$$
\begin{array}{|rrrr}
F & E & E^{2} & F^{2} \\
\hline 1 & 1 & 1 & 1 \\
1 & 1 & -1 & -1 \\
1 & 1 & -1 & -1 \\
1 & 1 & 1 & 1 \\
1 & -1 & 1 & -3 \\
-1 & 1 & 1 & -3 \\
1 & -1 & -3 & 1 \\
-1 & 1 & -3 & 1 \\
0 & 0 & -2 & -2 \\
0 & 0 & 2 & 2
\end{array}
$$

In particular, $\chi_{9}, \chi_{10}$ have height 1 , and by (5A)(iii) $\chi_{i}$ has height $\geqq 3$ for $i \geqq 11$; these $\chi_{i}$ then actually have height 3 or 4 by [4].

The columns $\mathfrak{D}^{E N}, \mathfrak{D}_{1}^{E N}+\mathfrak{D}_{2}^{E N}$ in cases II, III respectively are now completely determined from $\mathfrak{w}$ and $D^{F^{2}}$. In II the entries of $\grave{D}_{1}^{E N}, D_{2}^{E N}$ can be determined as follows: $\left(\mathfrak{D}_{1}^{E N}+\mathfrak{D}_{2}^{E N}\right)_{i}= \pm 1$ for $1 \leqq i \leqq 8,2$ for $i=9,10$. Since $\grave{D}_{\rho}^{E N}$ has norm 6 , the nonzero entries are \pm 1 . The product $\mathfrak{u D}_{1}^{E N}=2$ shows that one of $\left(D_{1}^{E N}\right)_{2}$, $\left(D_{1}^{E N}\right)_{3}$ is -1 and the other is 0 , since $\left(D_{1}^{E N}\right)_{10}=\left(D_{2}^{E N}\right)_{10}=1$. Since $\chi_{2}, \chi_{3}$ may be interchanged at this point, we may assume $\left(D_{1}^{E N}\right)_{2}=-1$. From the equality $\mathfrak{b D}_{1}^{E N}=0$, we have $\left(\mathfrak{D}_{1}^{E N}\right)_{3}=\left(\mathfrak{D}_{1}^{E N}\right)_{4}=0$. This completely determines the entries under $E N$ except for the arrangement $\left(\mathcal{D}_{1}^{E N}, \mathfrak{D}_{2}^{E N}\right)_{i}$ for $5 \leqq i \leqq 8$. Here $(1,0)$ can occur for $i=5$ and 8 , or for $i=6$ and 7 ; the remaining entries are $(0,1)$. This point will be settled below.

Let $s_{i}=(\mathfrak{S})_{i}$. Then $\mathfrak{S b}=-1, \mathfrak{S} \mathfrak{u}=1$ imply that $s_{2}+s_{3}+s_{4}=-1$, $-s_{2}-s_{3}+s_{10}=1$, so that $s_{4}=-s_{10}$. Also $\mathfrak{S a}=1$, $\mathfrak{G} \mathfrak{A}=1$ imply that $s_{5}=s_{8}$ and $s_{7}-s_{6}=1$. In particular, one of the numbers $s_{6}, s_{7}$ is \pm 1 , and the other is 0 . The quadratic form $Q^{J}$ is $10 u^{2}+2 v^{2}+(u-v)^{2}$. The method of contribution then implies the following: If $\chi_{i}$ has height 0 , then $\left(D_{1}^{J}, D_{2}^{J}\right)_{i}$, up to a sign change, can only be $(1,0),(01),(1,2),(-1,2),(0,3)$, and for $5 \leqq i \leqq 8$, only the first three are possible. If $\chi_{i}$ has height 1 , then only $(1,1),(0,2)$ are possible; if $\chi_{i}$ has height 3 , only $(1,3)$ is possible. If $\chi_{i}$ has height 4 , the method of contribution yields a contradiction. Since $s_{i}=0$ or \pm 1 , the definition of $\mathfrak{S}$ forces the conclusion $\left(D_{1}^{J}, D_{2}^{J}\right)_{i}=(1,0)$ for $i=5$ and 8 ; thus $s_{5}=s_{8}=0$. Similarly, $\left(D_{1}^{J}, D_{2}^{J}\right)_{i}=(0,1)$ or $(-1,-2)$ for $i=6,7$, and the latter can occur only in III by the method of contribution. Thus, if $s_{6}=-1, s_{7}=0$, then $\left(\mathfrak{D}_{1}^{J}, \searrow_{2}^{J}\right)_{i}=(0,1)$; if 
$s_{6}=0, s_{7}=1$, then $\left(D_{1}^{J}, D_{2}^{J}\right)_{i}=(-1,-2)$. We call these two possibilities IIIa, IIIb respectively. Suppose $s_{4}=0$. Then one of $s_{2}, s_{3}= \pm 1$, and the other is 0 . There must then be characters $\chi_{11}, \chi_{12}$ in $B$ such that $s_{11} \neq 0, s_{12} \neq 0$. For $i=11,12,\left(D_{1}^{J}, D_{2}^{J}\right)_{i}= \pm(1,3)$, and since $D_{1}^{J}$ has norm $6,\left(D_{1}^{J}\right)_{i}=0$ for $i=9$ or 10 . But this contradicts the fact that $\delta_{2}^{J}$ has norm 22. Thus $s_{4} \neq 0$, and we easily see that $s_{4}=-1, s_{10}=1, s_{2}=s_{3}=0 . s_{9}$ can be found from $\mathfrak{S w}^{1}=-1$. If $s_{9}=0$, $B$ contains one more character $\chi_{11}$ for which $s_{11}=1$. The matrix of decomposition numbers can now be completed, the uncertainty remaining in II under $E N$ being settled from $\delta_{1}^{J} \delta_{1}^{E N}=0$. We omit the details.

The complete matrices are in cases II, IIIa, IIIb respectively

\begin{tabular}{|rrrr|rr|rr|rrr|}
$F$ & $E$ & $F^{2}$ & \multicolumn{1}{c}{$E^{2}$} & \multicolumn{1}{c}{$E N$} & \multicolumn{1}{c}{$\boldsymbol{l}$} \\
\hline 1 & 1 & 1 & 1 & 1 & 0 & 1 & 0 & 1 & 0 & 0 \\
1 & 1 & -1 & -1 & -1 & 0 & 0 & -1 & 0 & 2 & 1 \\
1 & 1 & -1 & -1 & 0 & -1 & 0 & -1 & -1 & -1 & -1 \\
1 & 1 & 1 & 1 & 0 & 1 & -1 & 2 & 0 & -1 & 0 \\
1 & -1 & -3 & 1 & 0 & 1 & 1 & 0 & 0 & -1 & 0 \\
-1 & 1 & -3 & 1 & 1 & 0 & 0 & 1 & 0 & 0 & 1 \\
1 & -1 & 1 & -3 & 1 & 0 & 0 & 1 & 0 & 0 & 1 \\
-1 & 1 & 1 & -3 & 0 & 1 & 1 & 0 & 0 & -1 & 0 \\
0 & 0 & -2 & -2 & 1 & 1 & -1 & -1 & 0 & 1 & -1 \\
0 & 0 & 2 & 2 & 1 & 1 & 0 & -2 & -1 & 1 & 0 \\
0 & 0 & 0 & 0 & 0 & 0 & -1 & -3 & 1 & -2 & 1 \\
\hline
\end{tabular}

The matrix of Cartan invariants of $B$ is

$$
\left[\begin{array}{rrr}
4 & -2 & 2 \\
-2 & 14 & 0 \\
2 & 0 & 6
\end{array}\right) \text {. }
$$

If $f_{1}, f_{2}, f_{3}$ are the degrees of the corresponding basic set, then $f_{1}=1, f_{2} \equiv-9$ $(\bmod 32), f_{3} \equiv-11(\bmod 32)$.

\begin{tabular}{|rrrr|r|rr|rrrr|}
$F$ & $E$ & \multicolumn{1}{c}{$F^{2}$} & \multicolumn{1}{c}{$E^{2}$} & \multicolumn{1}{c}{$\boldsymbol{E} N$} & \multicolumn{1}{c|}{1} \\
\hline 1 & 1 & 1 & 1 & 1 & 1 & 0 & 1 & 0 & 0 & 0 \\
1 & 1 & -1 & -1 & -1 & 0 & -1 & 0 & 1 & 2 & 1 \\
1 & 1 & -1 & -1 & -1 & 0 & -1 & -1 & -1 & -1 & -1 \\
1 & 1 & 1 & 1 & 1 & -1 & 2 & 0 & 0 & -1 & 0 \\
1 & -1 & -3 & 1 & 1 & 1 & 0 & 0 & 0 & -1 & 0 \\
-1 & 1 & -3 & 1 & 1 & 0 & 1 & 0 & 0 & 0 & 1 \\
1 & -1 & 1 & -3 & 1 & 0 & 1 & 0 & 0 & 0 & 1 \\
-1 & 1 & 1 & -3 & 1 & 1 & 0 & 0 & 0 & -1 & 0 \\
0 & 0 & -2 & -2 & 2 & -1 & -1 & 0 & 0 & 1 & -1 \\
0 & 0 & 2 & 2 & 2 & 0 & -2 & -1 & 0 & 1 & 0 \\
0 & 0 & 0 & 0 & 0 & -1 & -3 & 1 & 0 & -2 & 1 \\
\hline
\end{tabular}


The matrix of Cartan invariants is

$$
\left[\begin{array}{rrrr}
4 & 1 & -2 & 2 \\
1 & 2 & 3 & 2 \\
-2 & 3 & 14 & 0 \\
2 & 2 & 0 & 6
\end{array}\right\} .
$$

$f_{1}=1, f_{2} \equiv 32(\bmod 64), f_{3} \equiv 7(\bmod 64), f_{4} \equiv-11(\bmod 32)$.

\begin{tabular}{rrrrr|r|rr|rrr|}
$F$ & $E$ & $F^{2}$ & $E^{2}$ & \multicolumn{1}{c}{$E N$} & \multicolumn{1}{c}{$J$} \\
\hline 1 & 1 & 1 & 1 & 1 & 1 & 0 & 1 & 0 & 0 \\
1 & 1 & -1 & -1 & -1 & 0 & -1 & 0 & 1 & -1 \\
1 & 1 & -1 & -1 & -1 & 0 & -1 & 0 & -1 & -1 \\
1 & 1 & 1 & 1 & 1 & -1 & 2 & -1 & 0 & 2 \\
1 & -1 & -3 & 1 & 1 & 1 & 0 & -1 & 0 & 2 \\
-1 & 1 & -3 & 1 & 1 & -1 & -2 & 0 & 0 & 1 \\
1 & -1 & 1 & -3 & 1 & -1 & -2 & 0 & 0 & 1 \\
-1 & 1 & 1 & -3 & 1 & 1 & 0 & -1 & 0 & 2 \\
0 & 0 & -2 & -2 & 2 & 0 & 2 & 1 & 0 & -3 \\
0 & 0 & 2 & 2 & 2 & 0 & -2 & 0 & 0 & -2 \\
\hline
\end{tabular}

The Cartan matrix is

$$
\left[\begin{array}{rrr}
5 & 0 & -9 \\
0 & 2 & 0 \\
-9 & 0 & 29
\end{array}\right) \text {. }
$$

$f_{1}=1, f_{2} \equiv 0(\bmod 32), f_{3} \equiv 29(\bmod 64)$.

6. We now use the methods of [2] to complete the investigations of cases II and III. We recall the following facts: Let $\mathfrak{5}$ be a finite group of even order $g$. Let $p$ be a prime, and $P$ a $p$-element of $(\mathfrak{5}$. Let $\tilde{\mathfrak{G}}$ be a subgroup of order $\tilde{g}$ such that $\tilde{\mathfrak{G}} \geqq \mathfrak{C}^{*}(P)=\left\langle G \in \mathfrak{G} \mid G^{-1} P G=P^{ \pm 1}\right\rangle$. If $J_{\alpha}$ and $J_{\beta}$ are two involutions of $\mathbb{G}$, and $\left\{\phi_{\rho}^{P}\right\}$ is a basic set for a $p$-block of $\mathfrak{C}(P)$, then

$$
g \sum \chi_{\mu}\left(J_{\alpha}\right) \chi_{\mu}\left(J_{\beta}\right) d_{\mu \rho}^{P} / x_{\mu}=\tilde{g} c\left(J_{\alpha}\right) c\left(J_{\beta}\right) \sum h_{\mu \alpha} h_{\mu \beta} \tilde{d}_{\mu \rho}^{P} / \tilde{x}_{\mu} .
$$

Here on the left $\chi_{\mu}$ runs over the irreducible characters of the block $B$ of $(5$ corresponding to the block $b$ of $\mathfrak{C}(P), x_{\mu}=\chi_{\mu}(1)$, and $d_{\mu \rho}^{P}$ are the corresponding generalized decomposition numbers. On the right, $h_{\mu \alpha}=\Sigma_{\lambda} \tilde{\chi}_{\mu}\left(\tilde{J}_{\alpha, \lambda}\right) / \tilde{c}\left(\tilde{J}_{\alpha, \lambda}\right), \tilde{\chi}_{\mu}$ runs over characters of the block $\tilde{B}$ of $\tilde{\mathfrak{G}}$ corresponding to $b, \tilde{x}_{\mu}=\tilde{\chi}_{\mu}(1), \tilde{d}_{\mu \rho}^{P}$ are the corresponding generalized decomposition numbers, and $\left\{\tilde{J}_{\alpha, \lambda}\right\}$ ranges over a set of representatives of the conjugate classes of $\tilde{\mathfrak{F}}$ contained in the conjugate class of $J_{\alpha}$ in $\left(5\right.$. For notational convenience, we will let $L\left(J_{\alpha}, J_{\beta}, \grave{D}_{\rho}^{P}\right), R\left(J_{\alpha}, J_{\beta}, \widehat{D}_{\rho}^{P}\right)$ denote the left-hand side, the right-hand side of (6.1) respectively. 
In the applications to follow, we will always take $p=2$ and $\mathfrak{b}$ the 1-block of $\mathfrak{C}(P)$. It then follows that $B$ and $\widetilde{B}$ are the 1-blocks of $\mathbb{G}$ and $\tilde{\mathfrak{G}}$ respectively. As a second remark, we note that if $\tilde{\mathfrak{F}}$ has a normal 2-complement, then the characters $\tilde{\chi}_{\mu}$ in the 1-block of $\tilde{\mathfrak{F}}$ can be identified with the irreducible characters $\theta_{\mu}$ of an $S_{2}$-subgroup $\mathfrak{Q}$ of $\tilde{\mathfrak{G}}$. Moreover, if $J_{\alpha}, J_{\beta}, P$ are in $\mathfrak{Q}$, then $c\left(J_{\alpha}\right)^{-1} c\left(J_{\beta}\right)^{-1} R\left(J_{\alpha}, J_{\beta}, D_{1}^{P}\right)$ is equal to

$$
\begin{aligned}
\tilde{g} \sum_{\mu} \sum_{\lambda} \frac{\theta_{\mu}\left(\tilde{J}_{\alpha, \lambda}\right)}{\tilde{c}\left(\tilde{J}_{\alpha, \lambda}\right)} \sum_{v} \frac{\theta_{\mu}\left(\tilde{J}_{\beta, v}\right)}{\tilde{c}\left(\tilde{J}_{\beta, v}\right)} \frac{\tilde{d}_{\mu}^{P}}{\theta_{\mu}(1)} \\
\quad=\tilde{g} \sum_{\lambda, v} \sum_{\mu} \frac{\theta_{\mu}\left(\tilde{J}_{\alpha, \lambda}\right) \theta_{\mu}\left(\tilde{J}_{\beta, v}\right) \theta_{\mu}(P)}{\theta_{\mu}(1) c_{Q}\left(\tilde{J}_{\alpha, \lambda}\right) c_{Q}\left(\tilde{J}_{\beta, v}\right)} \frac{c_{Q}\left(\tilde{J}_{\alpha, \lambda}\right) c_{Q}\left(\tilde{J}_{\beta, v}\right)}{\tilde{c}\left(\tilde{J}_{\alpha, \lambda}\right) \tilde{c}\left(\tilde{J}_{\beta, v}\right)} \\
=(\tilde{\mathfrak{G}}: \mathbb{Q}) \sum_{\lambda, v} a_{Q}\left(\tilde{J}_{\alpha, \lambda}, \tilde{J}_{\beta, v}, P\right) \frac{c_{\Omega}\left(\tilde{J}_{\alpha, \lambda}\right) c_{Q}\left(\tilde{J}_{\beta, v}\right)}{\tilde{c}\left(\tilde{J}_{\alpha, \lambda}\right) \tilde{c}\left(\tilde{J}_{\beta, v}\right)}
\end{aligned}
$$

where $a_{\mathbb{Q}}\left(\tilde{J}_{\alpha, \lambda}, \tilde{J}_{\beta, v}, P\right)$ is the multiplicity of the class sum of $P$ in $\mathbb{Q}$ in the product of the class sums of $\tilde{J}_{\alpha, \lambda}$ and $\tilde{J}_{\beta, v}$. We assume in this that the elements $\tilde{J}_{\alpha, \lambda}$ and $\tilde{J}_{\beta, v} \in \mathbb{Q}$. Finally, we note that (6.1) can be added over linear combinations of the $d_{\mu \rho}^{P}$ for varying $\tilde{\mathfrak{G}}, P$, and $\rho$. If $\mathfrak{y}$ is such a combination of the $\mathfrak{D}_{\rho}^{\boldsymbol{P}}$ we understand by the equality $L\left(J_{\alpha}, J_{\beta}, \mathfrak{y}\right)=R\left(J_{\alpha}, J_{\beta}, \mathfrak{y}\right)$ the result obtained by first applying (6.1) to the various $\mathfrak{D}_{\rho}^{P}$, and then summing appropriately. We now turn to the situation at the end of $\S 5$.

Case III. Set $\beta=f_{2}^{J}, \beta \equiv-3(\bmod 32)$. If we set $\tilde{\mathfrak{G}}=\mathfrak{C}^{*}(F)$ and $\mathfrak{C}^{*}(E)$, and compute $L(J, J, \mathfrak{b})=R(J, J, \mathfrak{b})$, and $L(J, E N, \mathfrak{b})=R(J, E N, \mathfrak{b})$, we find that

$$
\begin{aligned}
& 1+\frac{\beta^{2}}{x_{2}}+\frac{\beta^{2}}{x_{3}}+\frac{(2 \beta-1)^{2}}{x_{4}}=0, \\
& 1+\frac{\beta}{x_{2}}+\frac{\beta}{x_{3}}+\frac{2 \beta-1}{x_{4}}=0 .
\end{aligned}
$$

Here the right-hand sides are computed by observing that $\mathfrak{C}^{*}(E), \mathfrak{C}^{*}(F)$ have normal 2-complements, and that $E, F$ do not occur in III in the appropriate product of involutions. Subtracting the second from the first, and cancelling $\beta-1$, which is permissible since $\beta \equiv-3(\bmod 32)$, we have

$$
\frac{\beta}{x_{2}}+\frac{\beta}{x_{3}}+2 \frac{2 \beta-1}{x_{4}}=0 \text {. }
$$

Substituting (6.4) into (6.3) then yields $1-(2 \beta-1) / x_{4}=0$, or $x_{4}=2 \beta-1$. Thus $J$ is in the kernel of $\chi_{4}$. By (6.3) $\beta / x_{2}+\beta / x_{3}+2=0$. Since $\chi_{2}(J)=\chi_{3}(J)$ $=-\beta$, and $|\beta| \leqq\left|x_{2}\right|,\left|x_{3}\right|$, we see that $x_{2}=x_{3}=-\beta$, and hence $J$ belongs to the kernel of $\chi_{i}$ for $1 \leqq i \leqq 4$.

In IIIb, $x_{2}=f_{2}-f_{3}, x_{3}=-f_{2}-f_{3}$; thus $f_{2}=0, f_{3}=\beta$. But $\chi_{6}(J)=-1-2 \beta$, and $x_{6}=\beta$. Since $|1+2 \beta| \leqq|\beta|$, we must have $\beta=-1$, which contradicts 
the congruence $\beta \equiv-3(\bmod 32)$. Thus IIIb is impossible. We henceforth assume we have IIIa. In this case, $\chi_{4}(J)=x_{4}$ implies that $f_{3}=1-2 \beta$.

If $\tilde{\mathscr{G}}=\mathfrak{C}^{*}(E N), \mathfrak{C}^{*}\left(F^{2}\right)$ respectively, and we compute $L(J, J, \mathfrak{w})=R(J, J, \mathfrak{w})$, then

$$
\frac{1}{x_{5}}+\frac{\beta^{2}}{x_{6}}+\frac{(1+\beta)^{2}}{x_{9}}=0 \text {. }
$$

Here the right-hand side is computed by noting that $\mathfrak{C}^{*}(E N), \mathfrak{C}^{*}\left(F^{2}\right)$ have normal 2-complements, and by using (6.2). If we substitute $x_{5}=-f_{3}, x_{6}=f_{4}, x_{9}=f_{3}-f_{4}$ into (6.5) and simplify, we obtain the equality $f_{4}+\beta f_{3}=0$, so that $f_{4}=2 \beta^{2}-\beta$. The equality $\chi_{2}(J)=x_{2}$ implies $f_{2}=-2(\beta-1)^{2}$. Summarizing, we have

$$
f_{1}=1, f_{2}=-2(\beta-1)^{2}, f_{3}=1-2 \beta, f_{4}=\beta(2 \beta-1) \text {. }
$$

Let $\Omega=\bigcap$ kernel of $\chi_{i}$ for $i=1,2,3,4$. Since $J \in \Re, \Re$ must contain the subgroup $\mathfrak{A}=\left\langle J, X, E F^{3}\right\rangle$ of $\mathfrak{P}$ generated by the elements of $\mathfrak{P}$ conjugate in $\mathfrak{b}$ to $J . \mathfrak{A}$ is abelian of type $(2,2,2)$. Since $F^{2}, N \notin \mathfrak{R}, \mathfrak{A}$ is an $S_{2}$-subgroup of $\mathfrak{R}$. Assume now that $\mathfrak{G}$ has no normal $2^{\prime}$-subgroups $\neq 1$. Let $\mathfrak{N}$ be a minimal normal subgroup of $\mathfrak{G}$ contained in $\mathfrak{R}$. Since $|\mathfrak{N}|$ is even, $\mathfrak{U} \leqq \mathfrak{N}$.

Suppose $\mathfrak{U} \neq \mathfrak{N}$. Since $\mathfrak{N}$ is characteristically simple and $\mathfrak{U}$ is an $S_{2}$-subgroup of $\mathfrak{N}$, it follows that $\mathfrak{N}$ is simple. Hence $\mathfrak{W}=\mathfrak{N}_{\mathfrak{R}}(\mathfrak{U}) / \mathfrak{C}_{\mathfrak{N}}(\mathfrak{A})$ has order $7 \cdot 3^{x}$ with $x=0$ or 1 . Clearly $\mathfrak{P}$ stabilizes $\mathfrak{W}$, so we may view $\mathfrak{P} / \mathfrak{A}$ as a group of operators of $\mathfrak{W}$. If $P \in \mathfrak{P}$ and $P \mathfrak{A}$ centralizes $\mathfrak{W}$, then $\mathfrak{B}$ leaves $\mathfrak{A} \cap \mathfrak{C}(P)$ invariant. Since $\mathfrak{U}$ is an irreducible $\mathfrak{W}$-group, it follows that $\mathfrak{A} \leqq \mathfrak{C}(P)$, so $P \in \mathfrak{P} \cap \mathfrak{C}(\mathfrak{U})=\mathfrak{U}$. Thus $\mathfrak{P} / \mathfrak{A}$ is faithfully represented on $\mathfrak{W}$. This is impossible, since $|\mathfrak{P}: \mathfrak{A}|=8$ and $|\mathfrak{W}|$ divides $3 \cdot 7$. Thus $\mathfrak{U}=\mathfrak{N}$.

Since $\mathfrak{A}$ is a self-centralizing normal subgroup of $\mathfrak{P}$, it follows that $\mathfrak{C}(\mathfrak{A})=\mathfrak{A} \times \mathfrak{D}$, where $\mathfrak{D}$ has odd order. Hence $\mathfrak{D}$ char $\mathfrak{C}(\mathfrak{A}) \triangleleft \mathfrak{G}$, so $\mathfrak{D}=1$. Thus $\mathbb{b} / \mathfrak{A}$ is isomorphic to a subgroup of $G L(3,2)$ of order $\equiv 0(\bmod 8)$ which has no subgroup of index 2 . Hence $\mathfrak{5} / \mathfrak{A} \simeq G L(3,2)$. The group $\left(\mathfrak{5}\right.$ exis $^{+}$and is unique by [11].

Case II. Set $\alpha=f_{2}^{E N}, \beta=f_{2}^{J} ; \alpha \equiv-3(\bmod 8), \beta \equiv-3(\bmod 32)$. If we take $\tilde{\mathfrak{G}}=\mathfrak{C}^{*}(E), \mathfrak{C}^{*}(F)$ and compute $L(J, J, \mathfrak{b})=R(J, J, \mathfrak{b})$, and $L(E N, E N, \mathfrak{b})$ $=R(E N, E N, \mathfrak{b})$, we find that

$$
\begin{aligned}
1+\frac{\beta^{2}}{x_{2}}+\frac{\beta^{2}}{x_{3}}+\frac{(2 \beta-1)^{2}}{x_{4}} & =0, \\
1+\frac{1}{x_{2}}+\frac{\alpha^{2}}{x_{3}}+\frac{\alpha^{2}}{x_{4}} & =0 .
\end{aligned}
$$

The right-hand sides are 0 by observing that $\mathfrak{C}^{*}(F), \mathfrak{C}^{*}(E)$ have normal 2-complements, and that in II, $E, F$ do not occur in the appropriate products of involutions. Expressing the degrees $x_{i}$ in terms of $1=f_{1}, f_{2}, f_{3}$ and simplifying, we get 


$$
\begin{gathered}
\alpha^{2}-\frac{f_{2}\left(1+f_{2}+f_{3}\right)}{2 f_{2}+f_{3}}=0, \\
\beta^{2}\left(f_{2}-\alpha^{2}+4\left(1+f_{2}+f_{3}\right)\right)-4 \beta\left(1+f_{2}+f_{3}\right)-\left(1+f_{2}+f_{3}\right)\left(f_{2}-1\right)=0,
\end{gathered}
$$

(6.6) and (6.7) can be used to eliminate $f_{3}$; a little manipulation then gives the equation

$$
f_{2}^{2}\left(\beta^{2}-\alpha^{2}\right)+2 \alpha^{2} f_{2}(\beta-1)^{2}+\alpha^{2}\left(\alpha^{2} \beta^{2}-(2 \beta-1)^{2}\right)=0 .
$$

If $\alpha^{2} \neq \beta^{2}$, then the above equation factors into $\left(\beta^{2}-\alpha^{2}\right)\left(f_{2}-\lambda\right)\left(f_{2}-\mu\right)=0$, where

$$
\lambda=\frac{\alpha-2 \alpha \beta-\alpha^{2} \beta}{\beta+\alpha}, \quad \mu=\frac{-\alpha+2 \alpha \beta-\alpha^{2} \beta}{\beta-\alpha} .
$$

If $\alpha^{2}=\beta^{2}$, then $\alpha=\beta$ from the congruences $\alpha \equiv-3(\bmod 8), \beta \equiv-3(\bmod 32)$. In this case, $f_{2}=-\left(\alpha^{2}+2 \alpha-1\right) / 2$.

If we take $\tilde{\mathfrak{G}}=\mathfrak{C}^{*}\left(F^{2}\right), \mathfrak{C}^{*}(E N)$, and compute $L(J, J, \mathfrak{w})=R(J, J, \mathfrak{w})$, we have

or

$$
\frac{g}{c(J)^{2}}\left[\frac{(1+\beta)^{2}}{f_{2}-f_{3}}-\frac{1}{f_{2}}+\frac{\beta^{2}}{f_{3}}\right]=\frac{-c\left(F^{2}\right)}{c\left(F^{2}, N\right)^{2}}
$$

$$
g=-c(J)^{2} \frac{c\left(F^{2}\right)}{c\left(F^{2}, N\right)^{2}} \frac{f_{2} f_{3}\left(f_{2}-f_{3}\right)}{\left(\beta f_{2}+f_{3}\right)^{2}}
$$

Apply now the methods at the beginning of this section to the situation $\mathbb{G}=\mathbb{C}(J)$, $\tilde{\mathfrak{G}}=\mathfrak{C}^{*}\left(F^{2}\right)$, and $L\left(X, X, \mathfrak{D}^{F^{2}}\right)=R\left(X, X, \mathfrak{D}^{F^{2}}\right)$. Using (4.7), we see that $L\left(X, X, \mathfrak{D}^{F^{2}}\right)$ $=2 c(J)(\beta+3)^{2} / \beta(\beta+1)$. On the other hand, in $\mathbb{C}(J) X$ is fused only to $E F$, and $(\operatorname{ccl}(X) \cup \operatorname{ccl}(E F))^{2}$ does not involve $F^{2}$. Thus $R\left(X, X, \mathfrak{D}^{F^{2}}\right)=0$. and we must have $\beta=-3$. This already shows that $\alpha \neq \beta$; otherwise, $f_{2}=-1$, whereas $f_{2} \equiv-9(\bmod 32)$. Hence $f_{2}=\lambda$ or $\mu$ of (6.9). If $f_{2}=\lambda$, then $\alpha-2 \alpha \beta-\alpha^{2} \beta \equiv 0$ $(\bmod \beta+\alpha)$, and $\alpha+\beta$ divides $\beta(1-\beta)^{2}$. If $f_{2}=\mu$, then $-\alpha+2 \alpha \beta-\alpha^{2} \beta \equiv 0$ $(\bmod \beta-\alpha)$, and $\alpha-\beta$ divides $\beta(1-\beta)^{2}$. In either case, $\alpha \pm 3$ divides 48 . Only a small number of possibilities can occur for $\alpha$, and since $f_{2} \equiv-9, f_{3} \equiv-11$ $(\bmod 32)$, a check of these shows that only the following solution exists:

$$
\alpha=5, \quad \beta=-3, f_{2}=55, f_{3}=-11 \text {. }
$$

The degrees of the characters in $B$ are then $1,55,55,55,11,11,99,45,66,54,120$. In particular, $\mathfrak{5}$ has a rational character $\chi_{6}$ of degree 11 faithful on $\mathfrak{P}$. Assume now that $\left(5\right.$ has no normal $2^{\prime}$-subgroups $\neq 1$. Then by a theorem of Schur's, the order $g$ of $\left(5\right.$ divides $2^{6} \cdot 3^{6} \cdot 5^{2} \cdot 7 \cdot 11$.

The characters in the 1-block of $\mathfrak{C}(J) /\langle J\rangle$ have degrees $1,1,3,3,3,3,3,3,6,2$ by (4.7). Let $\theta=\theta_{j}$, for some $j$ where $3 \leqq j \leqq 8$. If $\Re /\langle J\rangle$ is the kernel of $\theta$, then for a suitable choice of $j$, the only nontrivial elements of $\mathfrak{P} /\langle J\rangle$ in $\Re /\langle J\rangle$ are the elements in the class of $e^{2}$. Since $\theta$ is a rational character, $(\mathcal{C}(J): \Re)$ is of 
the form $2^{s} 3^{t}$, so that $\mathfrak{C}(J) / \Re$ is solvable. If we now choose $\theta$ so that the only nontrivial elements of $\mathfrak{P} /\langle J\rangle$ in the kernel $\Omega_{1} /\langle J\rangle$ of $\theta$ are the elements in the class of $f^{2}$, then a similar argument shows that $\mathbb{C}(J) / \mathcal{R}_{1}$ is solvable of order $2^{s^{\prime}} 3^{t^{\prime}}$. But $\mathfrak{R} \cap \Omega_{1} \cap \mathfrak{P}=\langle J\rangle$. It now follows that if $\mathfrak{M}$ is the maximal normal $2^{\prime}$-subgroup of $\mathfrak{C}(J)$, then $\mathfrak{C}(J) / \mathfrak{M}$ is solvable, and its order is of the form $2^{a} 3^{\mathfrak{b}}$. Since $\mathfrak{C}(J) / \mathfrak{M}$ has only one block for the prime 2 by [5], the order of $\mathfrak{C}(J) / \mathfrak{M}$ is then 192. Set $m=|\mathfrak{M}|$.

The values of $\alpha, \beta, f_{2}, f_{3}$, and (6.10) show that the order of $(5)$ is

$$
g=2^{5} \cdot 3^{3} \cdot 5 \cdot 11 m \frac{m}{c\left(F^{2}, N\right)} \frac{c\left(F^{2}\right)}{c\left(F^{2}, N\right)} .
$$

We note that $c\left(F^{2}, N\right) \mid c\left(F^{2}\right)$, and $c\left(F^{2}, N\right) \mid 64 m$, the latter being true, since no 3-element of $\mathfrak{C}(J) / \mathfrak{M}$ commutes with $N$. In particular, $3^{4} \nmid m$ since $g \mid 2^{6} \cdot 3^{6} \cdot 5^{2} \cdot 7 \cdot 11$.

Suppose $p$ divides $m$, where $p=7$ or 5 . Since $7^{2} \nmid g, 5^{3} \nmid g,(6.11)$ implies that $p \mid c\left(F^{2}, N\right)$. Thus there exists an element $Y$ in $\mathfrak{M} \cap \mathfrak{C}\left(F^{2}\right)$ of order $p$. In particular, by $(5.3) \chi_{6}(J Y)=\chi_{6}\left(F^{2} Y\right)=3$ and so $\chi_{6}(Y) \equiv 3(\bmod 4)$. Since $\chi_{6}(Y)$ can assume only the value 4 for $p=7$, and the values 6 or 1 for $p=5$, this is impossible. Thus $m \mid 27$ and $g \mid 2^{6} \cdot 3^{6} \cdot 5 \cdot 11$.

Let $S$ be an element in $\mathfrak{G}$ of order 11 , and let $\mathfrak{S}=\langle S\rangle$. If $\mathfrak{N}(\mathfrak{S})=\mathfrak{C}(\mathfrak{S})$, then (5 would have a normal 11-complement. In particular, $\mathfrak{S}$ would normalize an $S_{2}$-subgroup $\mathfrak{P}_{1}$ of $\mathfrak{G}$ and hence centralize the central involution of $\mathfrak{P}_{1}$, which is impossible. Thus $\mathfrak{N}(\mathfrak{S}) \neq \mathfrak{C}(\mathfrak{S})$. The order of $\mathfrak{N}(\mathfrak{S}) / \mathfrak{C}(\mathfrak{S})$ is necessarily a divisor $\neq 1$ of 10 . The index (G: $\mathfrak{N}(\mathfrak{S}))$ is then of the form $32 \cdot 5 \cdot 3^{\alpha}, 32 \cdot 3^{\alpha}$, or $64 \cdot 3^{\alpha}$, where $0 \leqq \alpha \leqq 6$. By Sylow's theorem, this index is $\equiv 1(\bmod 11)$, and thus must be $64 \cdot 3^{3}$. If $m \neq 1$, then there would exist an element $T$ of order 3 in $\mathscr{C}(S)$. Since $\chi_{6}(S T) \equiv \chi_{6}(T)(\bmod 11)$ and $\chi_{6}(S T)=0$, we would have $\chi_{6}(T) \equiv 0(\bmod 11)$ and this is impossible. Thus $m=1$.

We have now shown that $g=2^{6} \cdot 3^{3} \cdot 5 \cdot 11=95,040$. Suppose $(5$ has proper normal subgroups; among these choose $\mathfrak{N}$ minimal. If $\mathfrak{b} / \mathfrak{N}$ has even order, then there would exist an irreducible character $\neq 1$ in the 1 -block of $\mathfrak{K} / \mathfrak{N}$ which would then belong to the 1-block $B$ of $(\mathfrak{b}$. But this is impossible, since the nontrivial characters in $B$ are faithful. If $\mathfrak{b} / \mathfrak{N}$ has odd order, then $\mathfrak{P}$ is an $S_{2}$-subgroup of $\mathfrak{N}$. If $\mathfrak{N}$ has no subgroups of index 2 , then $\mathfrak{N}$ has necessarily two classes of involutions, and the preceding work shows that $\mathfrak{N}$ has order 95,040 . Hence $\mathfrak{N}$ is solvable, and $\mathfrak{N}=\mathfrak{P}$. which is impossible. Thus $\mathfrak{C}$ is simple. By a theorem of Stanton [10], $\mathfrak{5}$ must be the Mathieu group $\mathfrak{M}_{12}$. Summarizing the results of this paper, we have

THEOREM (6A). Let $\left(5\right.$ be a finite group of order $64 g^{\prime}$, where $g^{\prime}$ is odd. Suppose there is an element $F$ of order 8 in $(5$ such that $F$ is self-centralizing in some $S_{2}$-subgroup $\mathfrak{P}$, and $F$ is conjugate to its odd powers in $\mathfrak{P}$. Then one of the following possibilities hold: 
(a) (5 has a subgroup of index 2 .

(b) $(5$ has one class of involutions.

(c) If $\mathrm{O}_{2},(\mathfrak{G})$ is the maximal normal subgroup of $\mathfrak{5}$ of odd order, then $\left(\mathfrak{b} / O_{2},(\mathfrak{G}) \simeq \mathfrak{G}_{1344}\right.$ or $\mathfrak{M}_{12}$, where $\mathfrak{G}_{1344}$ is a uniquely specified nonsimple, nonsolvable group of order 1344 , and $\mathfrak{M}_{12}$ is the Mathieu group on 12 symbols.

COROLlaRY (6B). The only simple group satisfying the assumptions of (6A) and having more than one class of involutions is $\mathfrak{M}_{12}$.

\title{
REFERENCES
}

1. R. Brauer, A characterization of the characters of groups of finite order, Ann. of Math. (2) 57 (1953), 357-377.

2. - Investigations on groups of even order. I, Proc. Nat. Acad. Sci. U. S. A. 47 (1961), 1891-1893.

3. - Some applications of the theory of blocks of characters of finite groups. I, J. Algebra 1 (1964), 152-167.

4. R. Brauer and W. Feit, On the number of irreducible characters of finite groups in a given block, Proc. Nat. Acad. Sci. U. S. A. 45 (1959), 361-365.

5. P. Fong, On the characters of p-solvable groups, Trans. Amer. Math. Soc. 98 (1961). 263-284.

6. G. Frobenius, Über die Charaktere der mehrfach transitiven Gruppen, S-B. Preuss. Akad. Wiss. (Berlin) (1904), 558-571.

7. M. Hall and J. Senior, The groups of order $2^{n}(n \leqq 6)$, Macmillan, New York, 1964,

8. P. Hall and G. Higman, On the p-length of p-soluble groups, Proc. London Math. Soc, 6 (1956), 1-42.

9. I. Schur, Uber eine Klasse von endlichen Gruppen linearer Substitutionen, S-B. Preuss. Akad Wiss. (Berlin) (1905), 77-91.

10. R. Stanton, The Mathieu groups, Canad. J. Math. 3 (1951), 164-174.

11. W. J. Wong, A characterization of the Mathieu group M12, Math. Z. 84 (1964), 378-388.

12. H. Zassenhaus, The theory of groups, Chelsea, New York, 1949.

\author{
HARVARD UNIVERSITY, \\ Cambridge, Massachusetts \\ UNIVERSITY OF CALIFORNIA, \\ Berkeley, CALIFornia
}

